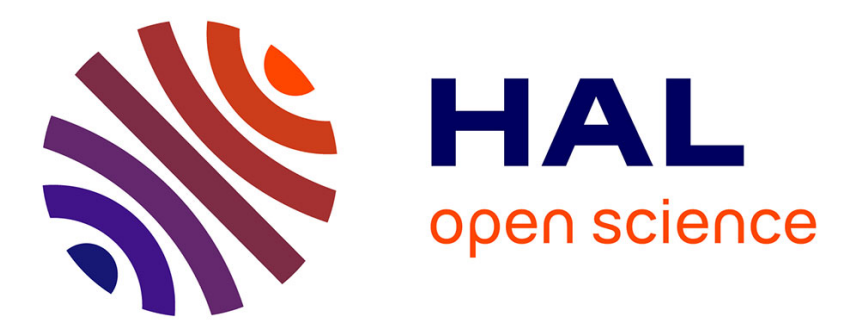

\title{
Reactive underwater object inspection based on artificial electric sense
}

\author{
Vincent Lebastard, Frédéric Boyer, Sylvain Lanneau
}

\section{To cite this version:}

Vincent Lebastard, Frédéric Boyer, Sylvain Lanneau. Reactive underwater object inspection based on artificial electric sense. Bioinspiration and Biomimetics, 2016, 11, pp.45003 - 45003. 10.1088/17483190/11/4/045003 . hal-01503252

\section{HAL Id: hal-01503252 \\ https://hal.science/hal-01503252}

Submitted on 6 Apr 2017

HAL is a multi-disciplinary open access archive for the deposit and dissemination of scientific research documents, whether they are published or not. The documents may come from teaching and research institutions in France or abroad, or from public or private research centers.
L'archive ouverte pluridisciplinaire HAL, est destinée au dépôt et à la diffusion de documents scientifiques de niveau recherche, publiés ou non, émanant des établissements d'enseignement et de recherche français ou étrangers, des laboratoires publics ou privés. 


\title{
Reactive underwater object inspection based on artificial electric sense
}

\author{
Vincent Lebastard, Frédéric Boyer, Sylvain Lanneau \\ La Chantrerie 4, rue Alfred Kastler, B.P. 20722, 44307 Nantes Cedex 3, France. \\ E-mail: Vincent.Lebastard@emn.fr.
}

May 2016

\begin{abstract}
The weakly electric fish can perform complex cognitive tasks based on extracting information from blurry electric images projected from their immediate environment onto their electro-sensitive skin. In particular they can be trained to recognize the intrinsic properties of objects such as their shape, size and electric nature. They do this by means of original perceptual strategies that exploit the relations between the physics of a self generated electric field, their body morphology and the ability to perform specific movement termed Probing Motor Acts (PMA). In this article we artificially reproduce and combine these PMA to build an autonomous control strategy that allows an artificial electric sensor to find electrically contrasted objects and to orbit around them based on a minimum set of measurements and simple reactive feedback control laws of the probe's motion. The approach does not require any simulation model and could be implemented on an Autonomous Underwater Vehicle (AUV) equipped with artificial electric sense. The AUV has only to satisfy certain simple geometric properties, such as bilaterally (left/right) symmetrical electrodes and possess a reasonably high aspect (length/width) ratio.
\end{abstract}

\section{Introduction}

In spite of many recent successes in underwater robotics, there is still no robot actually capable of operating in highly confined spaces in turbid waters [Lane, 2012]. These environments require manoeuvrability and sensing abilities that exceed the capacities of today's technologies. Though many efforts have been made in recent years to design new solutions for manoeuvrable underwater navigation from reconfigurable AUVs [Lane, 2012] to bio-mimetic fish like robots [Triantafyllou et al., 2000], the issue of sensing in these difficult conditions has had less attention from a research community that has traditionally been more oriented toward vision and sonar [Lane, 2012]. One promising approach takes inspiration from a group of fish species who posses "active electric sense". As Lissman and al. [Lissmann and Machin, 1958] discovered in the 50s, active electric fish perceive their immediate environment by measuring with a dense array of 
transcutaneous receptors, the perturbations of an electric field generated by an Electric Organ (EO) in the tail, that is polarized with respect to the rest of their body [von der Emde et al., 2012]. To use a visual metaphor, this field "lights" the close objects, projecting a dense electrical image of them on the skin which acts as an electric "retina" [von der Emde et al., 1998]. However, unlike vision, electric sense has no pre-reception mechanism comparable to the lens of the eye [Caputi, 2004]. The electric images projected onto the fish skin are fundamentally blurry and difficult to interpret [von der Emde et al., 2012]. Beyond these biophysical difficulties, a simple physical analysis of the electric responses of the polarized objects shows that their position and intrinsic geometry (shape, size) are intricately related in the measurement of the transcutaneous currents projected onto the skin [Rasnow, 1996]. Despite these difficulties, electric fish are able to perform complex cognitive tasks such as shape recognition [von der Emde, 1999]. One reason why the fish can do this is that they intensively move their bodies to extract useful information from these blurry images [Hofmann et al., 2013]. Electric fish have developed stereotyped locomotion patterns named probing motor acts (PMA) that they perform during object inspection [Toerring and Belbenoit, 1979, Toerring and Moller, 1984]. Three of these patterns are illustrated in figure 1. The first pattern (1) is named "stationary probing". In this behavior the fish approaches the object in straight line then stops suddenly when its head has reached a certain distance from it. The second behavior is named "lateral va et vient". This consists of forward and backward swimming movements at a constant distance from the object. The third behavior is termed "circling probing", which consists of a forward circling movement, whereby the fish swims at a constant distance around the object. As remarked in [Toerring and Moller, 1984], the characteristic distances observed in each of these three behaviors are independent of the object's intrinsic properties (shape, size and material).

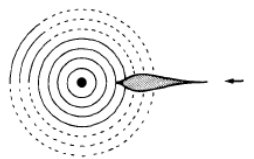

1

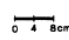

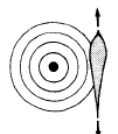

2

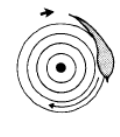

3

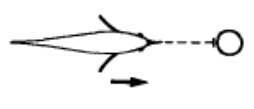

1

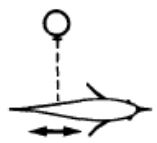

2

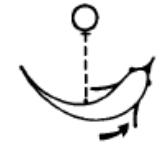

3

Figure 1. Probing Motor Acts (PMA) that fish use during object inspection: (1) Stationary probing. (2) Lateral "va et vient" probing. (3) Circling probing. (after Moller and Toerring [Toerring and Belbenoit, 1979, Toerring and Moller, 1984])

Since the 2000s a few roboticists have started to develop sensor technologies and algorithms inspired by electric fish with the aim of equipping a new generation of AUV capable of navigation and perception in constrained spaces and turbid waters [MacIver and Solberg, 2001, MacIver et al., 2004, Silverman et al., 2012, Porez et al., 2011, Lebastard et al., 2010, Boyer et al., 2012, Chevallereau et al., 2014]. Using various measurement principles [Silverman et al., 2012, Servagent et al., 2013], 
several robotics issues have been tackled from object localisation and recognition [Lebastard et al., 2010] to reactive navigation [Boyer et al., 2013, Boyer et al., 2015]. Though these first results are encouraging we are still far from equalling the fishes' performance. One reason for this is that compared to the fish, which can build dense electric images, our sensors can only provide a few measurements collected by a sparse array of electrodes [Servagent et al., 2013]. To overcome these limitations, one promising approach consists of coupling action and perception into low-level sensory loops able to reproduce the fish PMA with the aim of enriching the useful information available from a sparse receptor array.

This article describes attempts to reproduce the basic PMA illustrated in figure 1 using a motion-controlled sensor that mimics active electric sense. These PMA make use of a global fully autonomous strategy devoted to the inspection of electrically contrasted objects [Boyer and Lebastard, 2012]. The strategy begins with a primary behavior that consists of seeking any electrically contrasted object in an unknown scene, as introduced in [Boyer et al., 2013]. A stopping phase, inspired by the stationary probing act (1), a clearance phase inspired by the lateral "va et vient" probing (2) and a circling probing phase (3) are then described. The whole strategy is suited to the inspection of any electrically contrasted object in an unknown environment. Furthermore, it works in real time and does not require any prior model of the environment. This is essential since modelling the electric response of arbitrary shaped objects can only be performed with heavy finite elements numerical solvers which are fundamentally unsuited to the computational capabilities requirements of underwater robotics. We have presented the approach in [Boyer and Lebastard, 2012], but in a very empirical way, and with no reference to the PMA nor detailed analysis of the different behaviors. In the present paper, we further analyse each phase of the entire strategy using models, simulations and experiments. This exhaustive approach has produced further insights into each of the phases, and open new perspectives and issues for the application of artificial electric sense to underwater robotics.

The article is structured as follows. In section 2 we present our sensor technology. In section 3 the experimental set up on which our control strategy has been developed and assessed is described. In section 4, the electric model of the slender sensors in presence of small ellipsoidal objects is presented and assessed in section 5 . In section 6 a control strategy optimized for object inspection is presented. Experiments based on this control law are detailed in section 7. Section 8 offers a conclusion and perspectives for further research.

\section{Sensor}

The first sensing system inspired by electric sense was designed in [Solberg et al., 2008]. It was composed of four point electrodes, two of them being set under voltage, while 
the voltage between the other two was measured, supplying data to a particle filter algorithm designed to estimate the location of spherical objects. Since then, other sensor designs have been developed using either a $U-U$ [Bai et al., 2012] or $U-I$ [Boyer et al., 2012, Servagent et al., 2013] strategy. The first letter designates the emission (here a voltage $U$ ), the second, the measurement variable. In all cases, the sensor is an insulating axisymmetric shell on which a set of metal electrodes are arrayed with bilateral (left-right) symmetry. In both cases, the electric field is generated by setting a voltage on at least two electrodes in contact with the water. Though both techniques $(U-U$ and $U-I)$ share this common emission principle, in the $U-U$ mode, the other electrodes are paired floating potential electrodes between which the voltage is measured. While in the $U-I$ mode, all of the electrodes except the emitter are grounded, and the currents that flow across each of them are measured. A set of sensors based on the $U-I$ measurement principle, named "slender probes" from their high aspect ratio (length/thickness), have been presented in [Servagent et al., 2013] and are currently being used to study electric sense. The probes' macro-electrodes are rings or hemispheres numbered $\mathcal{E}_{i}(i=1,2 \ldots n)$ from the tail to the head. Figure 2 shows one of these probes with 3 macro-electrodes $\mathcal{E}_{i=1,2,3}$. Except for the emitter $\mathcal{E}_{1}$, located in the tail, each of $\mathcal{E}_{i}$ is divided into a pair of two identical left-right measurement electrodes electrically isolated and named $e_{i l}$ and $e_{i r}$ ( $l$ meaning "left" and $r$ meaning "right"), such that $\mathcal{E}_{i}=e_{i l} \cup e_{i r}$, for $i=1,2,3$. Electrode $\mathcal{E}_{3}$ is termed the "headmacro-electrode" or more concisely the "head-electrode", $\mathcal{E}_{2}$, the "neck-electrode", and $\mathcal{E}_{1}$, the "tail-electrode", or more simply "the emitter".
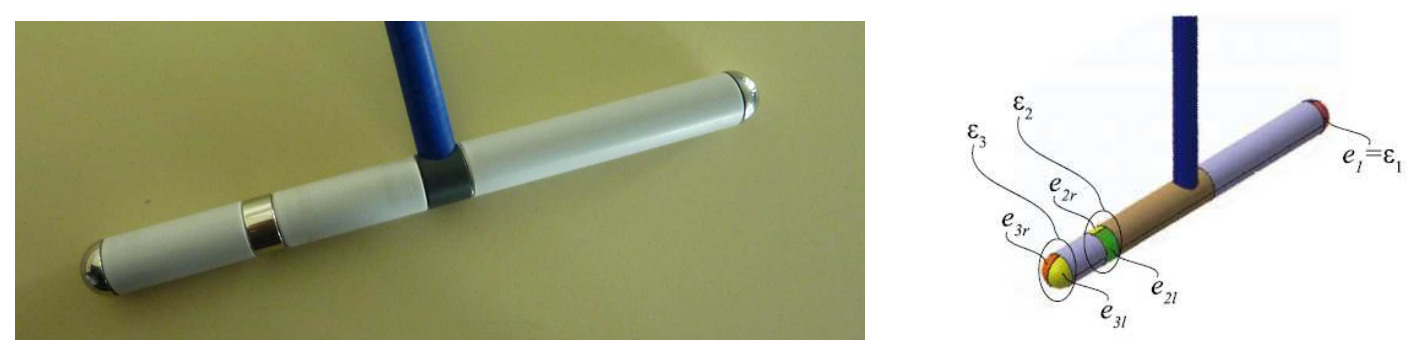

Figure 2. Photograph (left) and schematic view (right) of a 3-electrode sensor organized in 3 polarizable rings, 2 of them $\left(\mathcal{E}_{2}\right.$ and $\left.\mathcal{E}_{3}\right)$ being divided in two half rings $\left(e_{i l}, e_{i r}\right)$ allowing two lateral (left and right) current measurements.

The probe's overall length is $22 \mathrm{~cm}$, and its diameter is $2 \mathrm{~cm}$, yielding an aspect ratio $\simeq 10$. Using the standard electronics described in [Servagent et al., 2013], this sensor obtains a sensitivity of about $\pm 0.2 \%$ of the emitting current without object. In all the subsequent developments we used the sensor of figure 2 exploiting the head and neck electrodes. The vertical beam attached to the probe is used to control the motion of the probe as detailed in the next section. Once set under a voltage vector $\mathbf{U}=(U, 0,0)^{T}$ applied to $\left(\mathcal{E}_{1}, \mathcal{E}_{2}, \mathcal{E}_{3}\right)$, the response of the sensor with no object in the 
scene is given by the simple matrix model:

$$
\mathbf{I}^{(0)}=\mathbf{C}^{(0)} \mathbf{U}
$$

where $\mathbf{I}^{(0)}=\left(I_{1}^{(0)}, I_{2}^{(0)}, I_{3}^{(0)}\right)^{T}$ denotes the vector of the current flowing across $\mathcal{E}_{1}, \mathcal{E}_{2}$, and $\mathcal{E}_{3}$ respectively, while $\mathbf{C}^{(0)}$ is the conductance matrix of the scene with no object, which depends on the sensor's geometry and the surrounding water's conductivity, which is assumed to be homogeneous and equal to $\gamma_{o}$. Note that this matrix, which plays a crucial role in the subsequent inspection strategy, can be measured initially during a preliminary calibration or alternatively by using a (boundary) finite element (BEM) simulation. For instance, a BEM simulation for the 3-electrode probe in figure 2 yields the following expression:

$$
\mathbf{C}^{(0)}=\frac{\gamma_{o}}{100}\left(\begin{array}{ccc}
5.8162 & -2.9179 & -2.8680 \\
-2.9559 & 7.1193 & -4.2374 \\
-2.8602 & -4.2014 & 7.1054
\end{array}\right)
$$

where $\gamma_{o}$ is the conductivity of the surrounding water, which we assume to be homogeneous, isotropic and constant over time. Note that, due to the sensor's symmetries, the electric currents flowing across the sub-electrodes $e_{i l, r}$ are simply given by : $I_{2}^{(0)}=2 I_{2 r}^{(0)}=2 I_{2 l}^{(0)}$ and $I_{3}^{(0)}=2 I_{3 r}^{(0)}=2 I_{3 l}^{(0)}$ when there is no object in the scene and (1) contains all the information that the sensor can give in this case. Not also that we do not measure the current flowing across the emitter $\mathcal{E}_{1}$, but reconstruct it from the conservation law of currents $I_{1}=-I_{2}-I_{3}$. Finally, with our sensitivity, to recover the fish range which is about one and a half times the total body length [von der Emde, 1999], we need to set $U=5 \mathrm{~V}$, as this is done in all the following tests.

\section{Experimental setup}

An automated test bench has been built to test and validate our sensors and algorithms (see figure 3). This test bench comprises a tank of one cubic meter side with insulating walls and a three-axis cartesian robot (gantry). The tank is filled with tap water whose conductivity varies from 350 to $420 \mu \mathrm{S} / \mathrm{cm}$. The gantry is fixed on top of the tank and allows controlled translation along the $x$ and $y$ axes. The orientation in the $(x, y)$ plane can be controlled with a yaw-rotation stage. The three axes are motioncontrolled with the dSpace system using Matlab/Simulink software. The maximum speed is $0.3 \mathrm{~m} / \mathrm{s}$ for both $x$ and $y$ translations and $13.5 \mathrm{rpm}$ for rotation. The height of the sensor in the tank can be adjusted by using a rigid glass epoxy fiber tube. This vertical tube provides a conduit for the connecting wire that passes the signals coming from the electrodes to the electronic board. The signal is first amplified and filtered in the board's analog chain before passing to a 16 bit ADC Dspace card (DS2004) for digitization. The Dspace card (DS2004) can convert 16 channels simultaneously with a resolution of $0.3 \mathrm{mV} /$ bit (LSB) and a maximum conversion speed of 1.25 MHz. Our test bench is described in more detail in [Servagent et al., 2013]. 

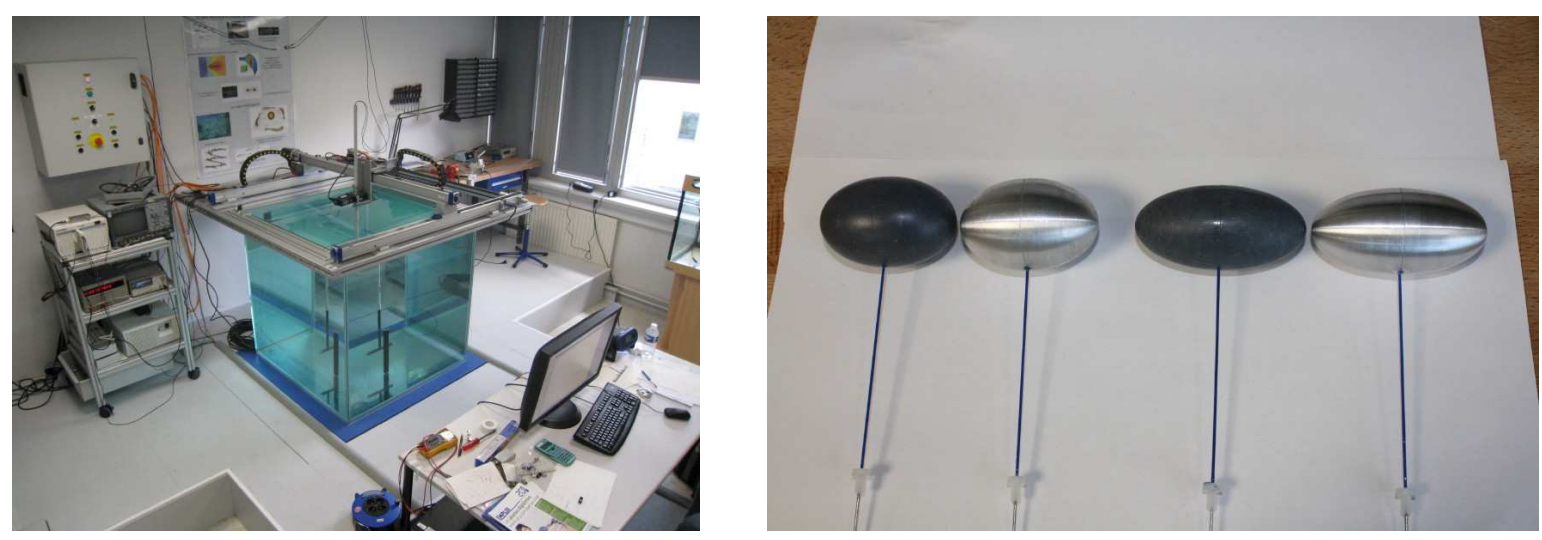

Figure 3. (left) Electrolocation test bench. (right) Example of a set of simple shaped objects (ellipsoids) of various sizes and materials (plastic, metal).

\section{Model}

We present a model for the control of a probe in this section. This includes a model of locomotion (section 4.1) and a model of electric perception (section 4.2). We will consider scenes in which a single small ellipsoidal object has been placed, and then more complex scenarios from experiments.

\subsection{Probe kinematic model and parameterization of a scene}

Throughout the article, we consider the probe described above, with its pose parameterized as indicated in figure 4 . The probe undergoes 2D motion in the horizontal plane. These motions are generated by a linear velocity $V$ parallel to the probe length and a yaw angular velocity $\Omega$ along the vertical axis. With these restrictions, the locomotion model of the probe can be modeled by the unicycle kinematics:

$$
\left(\begin{array}{c}
\dot{x} \\
\dot{y} \\
\dot{\beta}
\end{array}\right)=\left(\begin{array}{cc}
\cos \beta & -\frac{l \sin \beta}{2} \\
\sin \beta & \frac{l \cos \beta}{2} \\
0 & 1
\end{array}\right)\left(\begin{array}{l}
V \\
\Omega
\end{array}\right),
$$

where $(x, y, \beta)^{T}$ is the vector of Cartesian pose of a frame attached to the sensor head with respect to an inertial frame $\left(O, \mathbf{e}_{x}, \mathbf{e}_{y}\right)$ attached to the symmetry plane of the scene in which the probe moves (see figure $4(\mathrm{a})) .(V, \Omega)$ define the control inputs of the sensor, whose motions are modeled through (3). Note that in the case of an autonomous underwater vehicle (AUV), the above kinematic model can be used as a high control stage feeding a lower stage in charge of controlling the Cartesian velocities $(V, \Omega)$ through servo-propellers. The scene surrounding the probe will be assumed to be composed of a single small ellipsoidal object, whose pose is parameterized in the sensor mobile frame as indicated on figure 4(b) for our 3-electrode probe. 


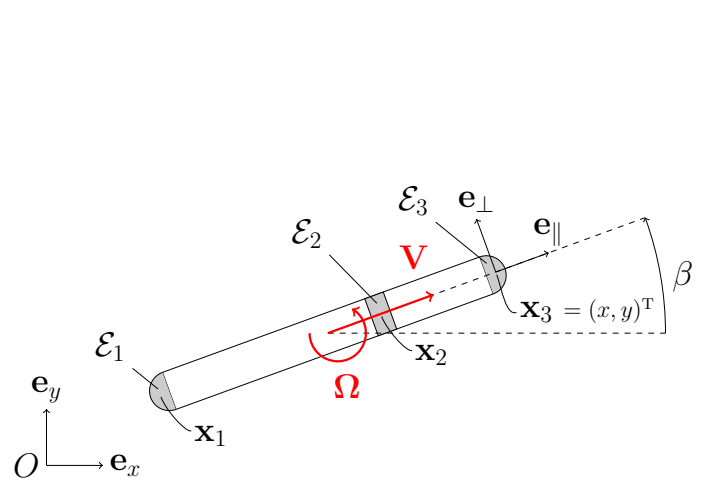

(a)

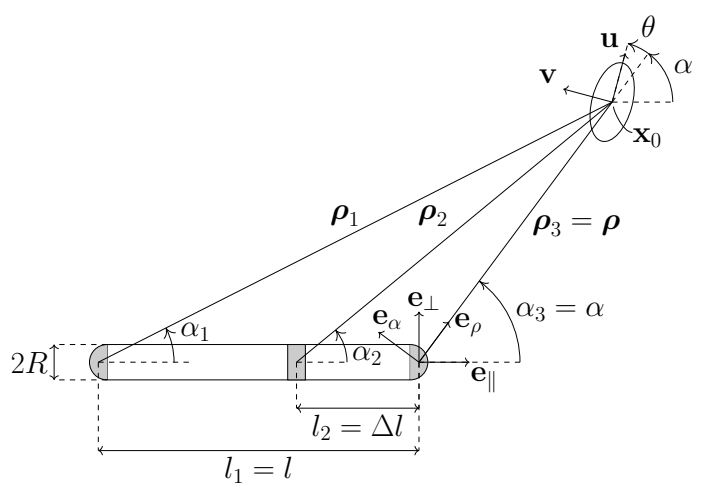

(b)

Figure 4. (a) Parametrization of a sensor pose , (b) and of a scene composed of an ellipsoidal small object.

Since the scene is at rest in the inertial frame, this (ego-centered) parameterization enables us to easily derive the following set of relations:

$$
\left(\begin{array}{c}
\dot{\rho} \\
\dot{\alpha} \\
\dot{\theta}
\end{array}\right)=\left(\begin{array}{cc}
-\cos \alpha & -\frac{l \sin \alpha}{2} \\
\frac{\sin \alpha}{\rho} & -\frac{l \cos \alpha}{2 \rho}-1 \\
-\frac{\sin \alpha}{\rho} & \frac{l \cos \alpha}{2 \rho}
\end{array}\right)\left(\begin{array}{c}
V \\
\Omega
\end{array}\right),
$$

defining the kinematic model of the scene with respect to the sensor frame.

\subsection{Model of electric measurement}

The model of the electric measurement of a $U-I$ sensor was introduced in [Boyer et al., 2012] for an arbitrary $n$-electrode slender probe. The key idea of [Boyer et al., 2012] consists in remarking first that the currents measured by the sensor, here our 3-electrode probe, are physically due to the electric equilibrium recovery of the sensor when it is unbalanced by an applied field of potential $\phi_{a}$. To derive the model of the electric sensor response, it is convenient to shift from the measured currents $I=\left(I_{l 1}, I_{r 1}, I_{l 2}, I_{r 2}, I_{l 3}, I_{r 3}\right)^{T}$ to the following set $(\mathrm{i}=1,2,3)$ :

$$
\begin{aligned}
& \delta I_{a x i}=\left(I_{i l}+I_{i r}\right)-I_{i}^{(0)}, \\
& \delta I_{\text {lati }}=I_{\text {lati }}=I_{i l}-I_{i r},
\end{aligned}
$$

where $\delta I_{a x i}$ and $\delta I_{\text {lati }}$ measured on $\mathcal{E}_{1}, \mathcal{E}_{2}$ and $\mathcal{E}_{3}$, are named the lateral and axial currents respectively.

\subsubsection{Model of the electric response of the sensor under an applied potential $\phi_{a}$}

Our probe's high aspect ratio allows the electric reaction to be decomposed into two components. As the electrokinetics are linear, they can be superimposed by addition to construct the total response (i.e. the measured currents crossing the receiver electrodes) of the sensor. As detailed in [Boyer et al., 2012], the first component 
is due to the polarization by $\phi_{a}$ of the sensor along its length and is termed "axial polarization". It is produced by the vector of potentials applied to all the macro-electrodes of the probe $\left(\mathcal{E}_{1,2,3}\right)$ :

$$
\boldsymbol{\Phi}_{a}=\left(\phi_{a}\left(\mathbf{x}_{1}\right), \phi_{a}\left(\mathbf{x}_{2}\right), \phi_{a}\left(\mathbf{x}_{3}\right)\right)^{T},
$$

where $\mathbf{x}_{i}$ generically denotes the position of the $\mathcal{E}_{i}$-electrode center (see figure 4 ). The second is due to the polarization by $\phi_{a}$ of the sensor along the direction perpendicular to its length and is termed "lateral polarization". This polarization is primarily generated by the following vector of axial excitation applied to the receiver electrodes $\left(\mathcal{E}_{1,2,3}\right)$ :

$$
\mathbf{E}_{a \perp}=-\left(\boldsymbol{\nabla} \phi_{a}\left(\mathbf{x}_{1}\right) \cdot \mathbf{e}_{\perp}, \nabla \phi_{a}\left(\mathbf{x}_{2}\right) \cdot \mathbf{e}_{\perp}, \nabla \phi_{a}\left(\mathbf{x}_{3}\right) \cdot \mathbf{e}_{\perp}\right)^{T} .
$$

where $\mathbf{e}_{\perp}$ is a unit vector perpendicular to the rostro-caudal axis of the sensor (figure 4(a)). The axial excitation (6) is comparable to the vector of voltages $\mathbf{U}$ imposed by the internal electronics, when all potentials $U_{i=1,2,3}$ are controlled independently as discussed in section 2. Thus, the currents produced by the axial polarization, gathered in the vector $\delta \mathbf{I}_{a x}=\left(\delta I_{a x 1}, \delta I_{a x 2}, \delta I_{a x 3}\right)^{T}$, are simply given by substituting $\mathbf{U}$ by $-\boldsymbol{\Phi}_{a}$ in the model of the sensor with no object (1):

$$
\delta \mathbf{I}_{a x}=-\mathbf{C}^{(0)} \boldsymbol{\Phi}_{a},
$$

where the minus sign indicates that the sensor has to oppose $\boldsymbol{\Phi}_{a}$ in order to recover its electric balance. The currents produced by the lateral polarization are gathered in the vector $\delta \mathbf{I}_{\text {lat }}=\left(\delta I_{\text {lat } 1}, \delta I_{\text {lat } 2}, \delta I_{\text {lat } 3}\right)^{T}$, modeled by the following relation:

$$
\delta \mathbf{I}_{l a t}=\mathbf{P}_{\perp} \cdot \mathbf{E}_{a \perp}
$$

where $\mathbf{P}_{\perp}$ is a tensor whose expression in the sensor frame is given by the diagonal matrix $P_{\perp}=\operatorname{diag}_{k=1,2,3}\left(p_{\perp i}\right)$, where the $p_{\perp i}$ are some "lateral polarizability factors" modeling the lateral distribution of the currents on the ring electrodes $\mathcal{E}_{i}$ when they are submitted to a (locally uniform) perpendicular electric field (here $-\boldsymbol{\nabla} \phi_{a} . \mathbf{e}_{\perp}$ ). In the following subsection we give the model of the applied potential $\phi_{a}$ when the sensor is positioned near to a small ellipsoidal object.

\subsubsection{Model of the potential $\phi_{a}$ generated by a small ellipsoidal object}

The purpose of this subsection is to present the model of the ambient field $\phi_{a}$ permitting the calculation of the measured currents $\delta \mathbf{I}_{a x}$ and $\delta \mathbf{I}_{l a t}$ when the probe is in presence of a small ellipsoidal object whose center is located in $\mathbf{x}_{o}$, using (6-9). Because the object is small, the potential produced by its polarization $\phi_{a}$, can be approximated by its leading order dipolar response in any point $\mathbf{x}$ of the scene as:

$$
\phi_{a}(\mathbf{x})=-\frac{\left(\mathbf{x}-\mathbf{x}_{o}\right) \cdot \mathbf{P} \cdot \nabla \phi^{(0)}\left(\mathbf{x}_{o}\right)}{\left\|\mathbf{x}-\mathbf{x}_{o}\right\|^{3}}
$$


where $\mathbf{p}=\mathbf{P} \cdot \boldsymbol{\nabla} \phi^{(0)}\left(\mathbf{x}_{o}\right)$ is the electric dipole generated by the polarization of the object by $-\boldsymbol{\nabla} \phi^{(0)}\left(\mathbf{x}_{o}\right) \triangleq \mathbf{E}_{0}$, i.e., the electric field in $\mathbf{x}_{o}$ that would be produced by the sensor should there be no object nearby. For any orientation of the incident field, this dipole is modeled by $\mathbf{P}$ which represents the dipolar tensor of the object. This is a linear operator intrinsically related to the object. Such a tensor has been calculated analytically for the case of an ellipsoid in [Ammari and Kang, 2007]. In an orthogonal frame $\left(O_{o}, \mathbf{u}, \mathbf{v}, \mathbf{w}\right)$ whose origin $O_{o}$ is located at the geometric center $\mathbf{x}_{o}$ of the ellipsoid, and with unit vectors $\mathbf{u}, \mathbf{v}, \mathbf{w}$ supporting the ellipsoid axes of half-length $a, b$ and $c$ respectively, the expression of the matrix of the components $P$ of $\mathbf{P}$ is:

$$
P=\left(\begin{array}{ccc}
\lambda_{u} & 0 & 0 \\
0 & \lambda_{v} & 0 \\
0 & 0 & \lambda_{w}
\end{array}\right)=\left(\gamma-\gamma_{o}\right) v\left(\begin{array}{ccc}
\frac{1}{\gamma_{o}+A\left(\gamma-\gamma_{o}\right)} & 0 & 0 \\
0 & \frac{1}{\gamma_{o}+B\left(\gamma-\gamma_{o}\right)} & 0 \\
0 & 0 & \frac{1}{\gamma_{o}+C\left(\gamma-\gamma_{o}\right)}
\end{array}\right)
$$

with $v$ the volume of the object, $\gamma$ the conductivity of its constitutive material, and where $A, B$ and $C$ are three geometric constants given by the following elliptic integrals:

$$
\begin{aligned}
A & =\frac{b c}{a^{2}} \int_{1}^{+\infty} \frac{1}{\xi^{2} \sqrt{\xi^{2}-1+\left(\frac{b}{a}\right)^{2}} \sqrt{\xi^{2}-1+\left(\frac{c}{a}\right)^{2}}} d \xi, \\
B & =\frac{b c}{a^{2}} \int_{1}^{+\infty} \frac{1}{\left(\xi^{2}-1+\left(\frac{b}{a}\right)^{2}\right)^{\frac{3}{2}} \sqrt{\xi^{2}-1+\left(\frac{c}{a}\right)^{2}}} d \xi, \\
C & =\frac{b c}{a^{2}} \int_{1}^{+\infty} \frac{1}{\sqrt{\xi^{2}-1+\left(\frac{b}{a}\right)^{2}}\left(\xi^{2}-1+\left(\frac{c}{a}\right)^{2}\right)^{\frac{3}{2}}} d \xi .
\end{aligned}
$$

These expression are valid for any small ellipsoidal object. In particular, for elongated (prolate) ellipsoid for which $a \geq b=c, A, B$ and $C$ take the simpler forms:

$$
\begin{aligned}
& A=\left(\frac{b}{a}\right)^{2} \int_{1}^{+\infty} \frac{1}{\xi^{2}\left(\xi^{2}-1+\left(\frac{b}{a}\right)^{2}\right)} d \xi \\
& B=\left(\frac{b}{a}\right)^{2} \int_{1}^{+\infty} \frac{1}{\left(\xi^{2}-1+\left(\frac{b}{a}\right)^{2}\right)^{2}} d \xi
\end{aligned}
$$

and $C=B$. When the object is made of an ideal conductive material such as metal $\gamma \gg \gamma_{o}$, and it is easy to show that $\lambda_{u}, \lambda_{v}, \lambda_{w}>0$. As a result, the object polarization tends to reinforce the ambient field $\phi^{(0)}$ while the electric current lines of the total field $\phi_{a}$ are funneled locally by the object. Meanwhile, when the object is made of an ideal insulating material (e.g. plastic or glass), $\gamma \ll \gamma_{o}$, and $\lambda_{u}, \lambda_{v}, \lambda_{w}<0$. In this case, the object produces a secondary field that opposes the ambient field, and the electric lines of the total field are repelled locally from the object boundaries. As shown in (10), the influence of the object on the sensor becomes comparable to that of the ambient field when its distance from the object is of the order of $\max (a, b, c)$. Furthermore, in the case of a sphere $a=b=c$, so $A=B=C=1 / 3$, and $P$ then reduces to the well known expression given in [Rasnow, 1996]:

$$
P=\chi a^{3} 1_{3 \times 3},
$$

with $1_{3 \times 3}$ the three order identity matrix, $a$, the sphere radius, and $\chi=\frac{\gamma-\gamma_{o}}{2 \gamma_{o}+\gamma}$ the so-called contrast factor of the sphere. We have chosen a small prolate ellipsoid 
with $a=2 b=2 \mathrm{~cm}$ as a test object in all that follows. Its major axis lies in the equatorial plane of the sensor. In these conditions, it is practical to introduce the following decomposition of $P$ :

$$
P=\bar{P}+\widehat{P}
$$

where $\bar{P}$ and $\widehat{P}$ define two further tensors, the first representing the isotropic response of the object, the second, its anisotropic response, each of them being easily deduced from $P$ through the following expressions:

$$
\begin{aligned}
& \bar{P}=\frac{1}{2}\left(\lambda_{u}+\lambda_{v}\right) 1_{3 \times 3} \triangleq \frac{1}{2} \bar{\lambda} 1_{3 \times 3}, \\
& \widehat{P}=\frac{1}{2}\left(\lambda_{u}-\lambda_{v}\right) 1_{3 \times 3} \triangleq \frac{1}{2} \hat{\lambda} 1_{3 \times 3} .
\end{aligned}
$$

\subsubsection{Model of the measured currents generated by a small ellipsoidal object}

We now have all the necessary ingredients to derive an analytical model of the currents measured by the probe in presence of an ellipsoid. By introducing (11), together with (15), into (10) we can compute the potential $\phi_{a}$ produced by the polarization of the object. When this is introduced into (6-9), we obtain the model of the six measured currents $(5)$ as $(\mathrm{i}=1,2,3)$ :

$$
\begin{aligned}
& \delta I_{\text {lati }}=\delta \bar{I}_{\text {lati }}+\delta \hat{I}_{\text {lati }}, \\
& \delta I_{\text {axi }}=\delta \bar{I}_{\text {axi }}+\delta \hat{I}_{\text {axi }},
\end{aligned}
$$

where the lateral and axial currents are both decomposed into two contributions, one (over-barred) produced by the isotropic part of $\mathbf{P}$, and the other (over-hatted), by its anisotropic part. After tedious but simple computation, we find the analytical expressions of the four (axial/lateral and iso/anisotropic) currents to be:

$$
\left.\begin{array}{l}
\delta \hat{I}_{\text {lati }} \\
\delta \bar{I}_{\text {lati }}
\end{array}\right\}=-\frac{p_{\perp i}}{4 \pi \gamma_{o} \rho_{i}^{3}} \sum_{k=1}^{3} \frac{I_{k}^{(0)}}{\rho_{k}^{3}}\left\{\begin{array}{l}
\hat{\lambda} \hat{f}_{i k}\left(\alpha, \theta, \rho, \alpha_{i}\right) \\
\bar{\lambda} \bar{f}_{i k}\left(\alpha, \theta, \rho, \alpha_{i}\right)
\end{array}\right.
$$

with

$$
\begin{aligned}
& \hat{f}_{i k}=l_{k}\left[3 \sin \left(\alpha_{i}\right) \cos \left(2(\theta+\alpha)-\alpha_{i}\right)-\sin (2(\theta+\alpha))\right]+ \\
& \quad \rho\left[3 \sin \left(\alpha_{i}\right) \cos \left(2 \theta+\alpha-\alpha_{i}\right)-\sin (2 \theta+\alpha)\right], \\
& \bar{f}_{i k}=3 l_{k} \sin \left(\alpha_{i}\right) \cos \left(\alpha_{i}\right)+\rho\left(3 \sin \left(\alpha_{i}\right) \cos \left(\alpha-\alpha_{i}\right)-\sin (\alpha)\right),
\end{aligned}
$$

and

$$
\left.\begin{array}{l}
\delta \hat{I}_{a x i} \\
\delta \bar{I}_{a x i}
\end{array}\right\}=-\frac{1}{4 \pi \gamma_{0}} \sum_{k, j=1}^{3} \frac{C_{i j}^{(0)} I_{k}^{(0)}}{\rho_{k}^{3} \rho_{j}^{3}}\left\{\begin{array}{l}
\hat{\lambda} \hat{g}_{j k}(\alpha, \theta, \rho) \\
\bar{\lambda} \bar{g}_{j k}(\alpha, \theta, \rho)
\end{array}\right.
$$

with

$$
\begin{aligned}
& \hat{g}_{j k}=l_{k} l_{j} \cos (2(\theta+\alpha))+\left(l_{k}+l_{j}\right) \rho \cos (2 \theta+\alpha)+\rho^{2} \cos (2 \theta), \\
& \bar{g}_{j k}=l_{k} l_{j}+\left(l_{k}+l_{j}\right) \rho \cos (\alpha)+\rho^{2} .
\end{aligned}
$$


In these expressions $\rho_{i}=\left\|\boldsymbol{\rho}_{i}\right\|=\left\|l_{i} \mathbf{e}_{\|}+\rho \mathbf{e}_{\rho}\right\|$, with $l_{1}=l, l_{2}=\Delta l$ and $l_{3}=0$. Referring to figure 4 , we have $\rho_{i} \sin \left(\alpha_{i}\right)=\rho \sin (\alpha)$ and $\rho_{i} \cos \left(\alpha_{i}\right)=\rho \cos (\alpha)+l_{i}$, all the other notations having been introduced above. As expected, contemplation of the above expressions shows that while the isotropic part of the currents (i.e. $\delta \bar{I}_{\text {lat }}$ and $\left.\delta \bar{I}_{a x}\right)$ only depend on the position of the object through the parameters $(\rho, \alpha)$, the anisotropic components $\left(\delta \hat{I}_{l a t}\right.$ and $\left.\delta \hat{I}_{a x}\right)$ depend both on the object's position and its orientation $\theta$. Similarly, removing $\widehat{\lambda}$ from the expressions above gives the model of the currents measured by the sensor in presence of a single sphere as was first introduced in [Boyer et al., 2013] for a 2-electrode probe.

\section{Evaluation of the analytical model}

Before addressing our control problem, let us assess the previous analytical model of measurements. To this end, we used the 3-electrode sensor illustrated in figure 2, together with two copies of our test ellipsoid, one insulating, the other conductive. Both were placed in the scene as illustrated in figure 5. The sensor is then translated

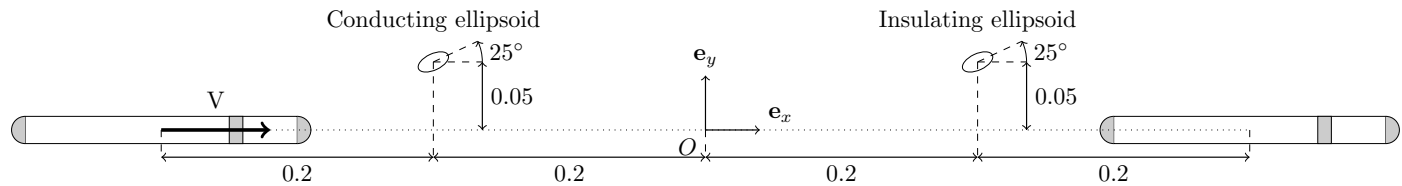

Figure 5. The 3-electrode probe is forward translated in the presence of one insulating and one conductive ellipsoid of same geometry $(a=0.012, b=0.006)$. Dimensions in meters.

through the scene along a rectilinear path while the currents that penetrate it are computed using both our BEM reference numerical simulator, and the analytical model described in the previous section. The results are displayed in figure 6 . The lateral and axial currents of (5) are plotted against the probe's position along its path.

A good match between the analytical model and the reference simulator was obtained. The reference simulator runs in-house code based on the Boundary Elements Method (BEM). It integrates the Laplace equations of the electric potential field without approximation of the boundary geometry (sensor and objects), except those unavoidably introduced by their finite-element meshing. The discrepancies observed in figure 6 are only due to the reduction process developed in [Boyer et al., 2012] which is essentially based on the method of reflections and the perturbation expansion of the Laplace equations with respect to the aspect ratio of the sensor. Finally, note that compared to a similarly sized conductive ellipsoid, the measured currents obtained for the insulating ellipsoid is negative and its magnitude is nearly half lower, which agrees with the findings in [Rasnow, 1996]. 

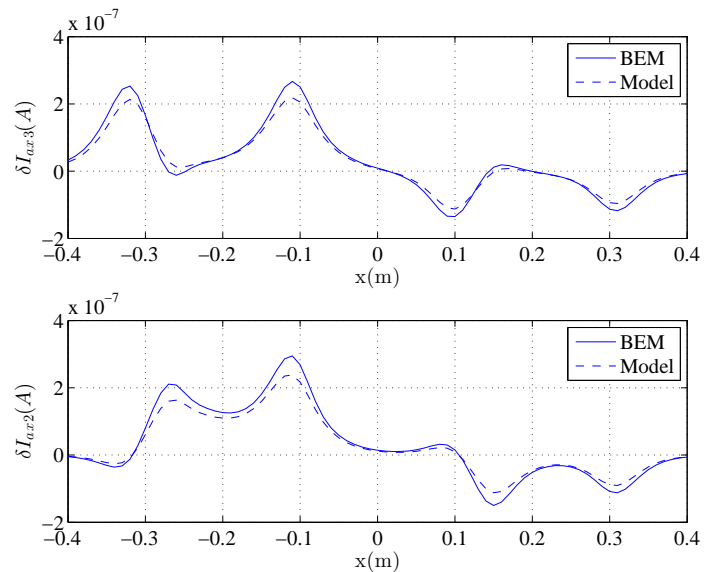

(a) Axial current versus the position of the probe along the path.
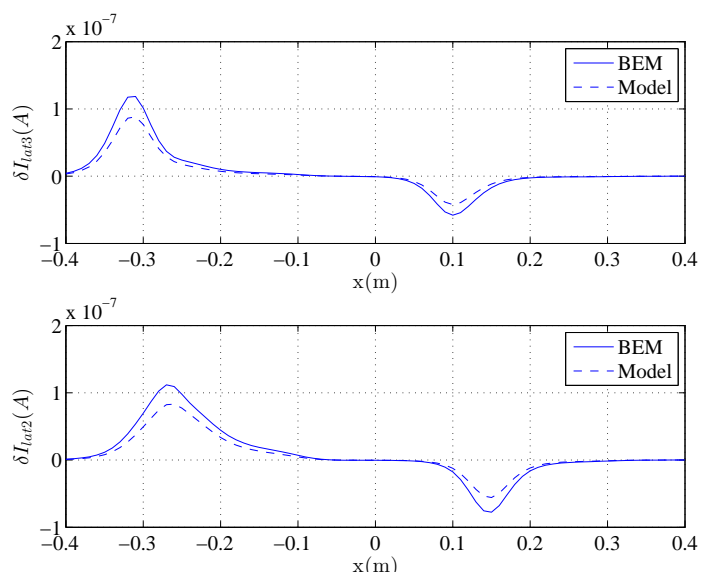

(b) Lateral current versus the position of the probe along the path.

Figure 6. (a) Axial and (b) lateral currents predicted by the analytical model and the BEM simulation with a 3-electrode sensor translated in the scene as pictured in figure 5 .

\section{Reactive control strategy}

In this section, we introduce a control strategy that allows the sensor to seek and inspect electrically contrasted objects in unknown scene. The strategy is presented in this section. Its physical interpretation then follows. Inspired by the fish PMAs outlined in section 1, by "sense and inspect" we here mean: to seek them and once discovered, to stop in front of them and to follow their boundaries without touching them. This strategy is achieved in a purely autonomous manner through the combination of three basic behaviors, co-ordinated according to the algorithm presented in figure 7(a). Its application to a small object is illustrated in figure 7(b). The sequence of the implemented behaviors is summarized as follows:

- 1) Seek any object that is electrically contrasted with respect to the ambient medium. This first phase (from $A$ to $B$ in figure 7 ) consists of seeking an electrically non-transparent object by applying an attractive control law with a constant forward velocity $V>0$.

- 2) Retreat from the electric influence of the object. This second phase (from $B$ to $B^{\prime}$ in figure 7) corresponds to the initialization of an orbiting motion of the sensor around the object. It is obtained by applying a repulsive control law with $V=0$ and $\Omega \neq 0$.

- 3) Follow the boundaries of the object. This third phase (from $B^{\prime}$ to $C$ in figure 7) stands for the orbiting phase around the object's boundaries with a constant 
forward velocity $V>0$.

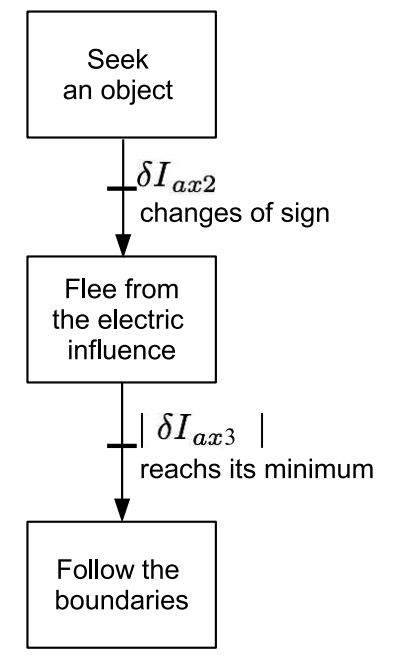

(a)

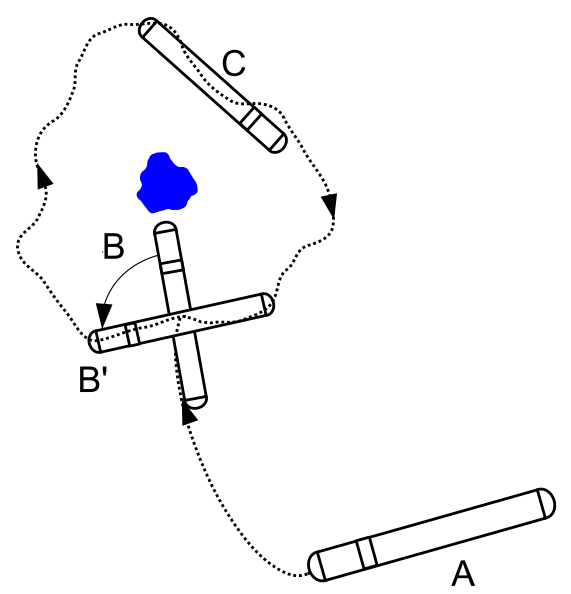

(b)

Figure 7. (a) Inspection task algorithm. (b) Typical object inspection scenario.

As we shall see below, all these behaviors can be achieved with our three-electrode probe. In particular, except for the tail emitter, which is required in all circumstances, the first and second phases require only the head electrode measurements $\left(\delta I_{a x 3}, \delta I_{\text {lat } 3}\right)$, while the third phase is based on the measurements of the neck electrode $\left(\delta I_{a x 2}, \delta I_{\text {lat } 2}\right)$. Moreover, as indicated by the algorithm in figure $7(\mathrm{a})$, the transition between the phases are governed by discrete events which depend only on the time variations of the measurements (and not their magnitude). The first transition from phase 1 to phase 2 , is activated by the change of sign of the axial current flowing across the neck electrode $\left(\delta I_{a x 2}\right)$. The second transition (from phase 2 to phase 3 ) is activated when the axial current flowing across the head-electrode $\left(\delta I_{a x 3}\right)$ reaches its minimum absolute value. In the subsequent section we physically interpret this strategy.

\subsection{Physical interpretation of the reactive strategy}

The above strategy can be easily explained in terms of interactions between the sensor and the electric field lines emitted by the objects in their immediate environment. In (7), note that the lateral current $\delta I_{\text {lat3 }}$ can be detailed as $\delta I_{l a t 3}=-p_{\perp 3} \boldsymbol{\nabla} \phi_{a}\left(\mathbf{x}_{3}\right) \cdot \mathbf{e}_{\perp}$ with $\phi_{a}$ given by (10). Thus, $\delta I_{\text {lat } 3}$ is proportional to the lateral flux of the electric field reflected by the object, and in the first phase, the attractive law which as we shall see later, forces $\delta I_{\text {lat } 3}=0$, also ensures the sensor aligns the front part of its body with the electric field lines of $\phi_{a}$, while following them at a constant velocity $V$. As a result, since all the electric lines of $\phi_{a}$ (the integral of the electric field reflected by the objects) emanate from the objects close to the sensor, the sensor is attracted by these objects. At the end of this first phase, as the sensor head gets closer and closer to, say a conductive object, the electric field lines originally (when there is no object around the sensor) captured by the neck electrode are attracted from the neck toward the head electrode. On the other hand, if the object is insulating the electric field 
lines are repelled backward along the sensor axis from the head to the neck electrode as illustrated in figure 8. In both cases a moment in time arrives at which $\delta I_{a x 2}$ changes sign. At that time the control law switches from the attractive behavior of phase 1 to the repulsive behavior of phase 2. When this occurs, the sensor starts to avoid the object while $V=0$, until $\left|\delta I_{a x 3}\right|$ reaches a minimum, which implies that the tail $\left(\mathcal{E}_{1}\right)$ and the neck and head electrodes $\left(\mathcal{E}_{2}, \mathcal{E}_{3}\right)$ are electrically equidistant from the object. If one of these two electrodes is closer to the object than the other, the electric lines emitted by the tail or the lines received by the head are more perturbed (than in the equidistant case) and the corresponding measurement $\left|\delta I_{a x 3}\right|$ is larger (see figure 8). When this condition is satisfied, $\delta I_{a x 3}$ is memorized as a reference value that the sensor tries to maintain by steering its body's axis while maintaining a constant forward velocity, thus maintaining an iso current path around the object's boundaries.
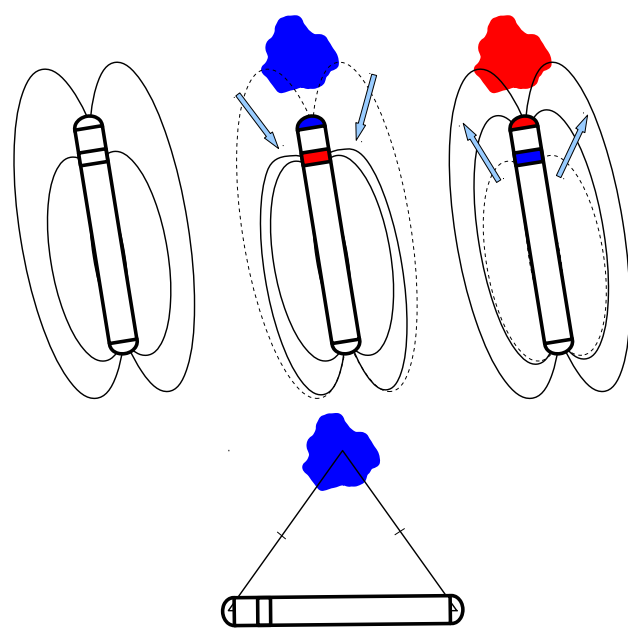

Figure 8. Physical interpretation of the reactive strategy in terms of electric field lines: (top) The electric lines are pushed forward or backward depending upon whether if the object facing the sensor is a conductor or an insulator. (bottom) When the head-neck and tail electrodes are electrically equidistant to the object, the head current reaches a minimum.

In the subsequent sections, we analyse each of these phases and transitions using the above analytical model, the BEM simulations, and the results from our experimental setup presented in section 3 .

\subsection{First phase: seek any electrically contrasted object}

In this subsection, we analyse the behavior "Seek any electrically contrasted object". This basic behavior is introduced in [Boyer et al., 2013] where it was achieved through the simple feedback law:

$$
V=V_{d}, \text { and: } \Omega=k \frac{\delta I_{\text {lat } 3}}{\left|\delta I_{a x 3}\right|} .
$$

Expression (24) ensures the attraction of the probe toward electrically contrasted objects of any shape and size. This was shown in [Boyer et al., 2013] where the probe's closed loop dynamics were analysed with an analytical model of the response of spherical objects. Here, we start from this simple case and advance the analysis to the more 
general case of ellipsoidal objects. In $(24), V_{d}$ is a constant positive value ensuring the forward motion of the probe with a constant axial velocity $V_{d} \mathbf{e}_{\|}$and $k$ is a steering gain which ensures the probe is attracted or repelled by any electrically contrasted object depending on whether $k$ is positive or negative respectively. Introducing the control law (24) in the kinematic model of the scene (4), and using the model of the electric currents (18-21), gives the closed-loop dynamics of the probe in the presence of a single small ellipsoidal object in the form $x^{\prime}=f(\xi, x)$ with $x=(\rho, \alpha, \theta)$, the state vector, and ' the derivation $d . / d s$ with $s=V t$ the length travelled by the probe along its path in the scene. In the simple case of spherical objects, the symmetry of the object ensures the closed loop-dynamics to take the simpler form $x^{\prime}=f(\xi, x)$ with $x=(\rho, \alpha)$. Thus, for a given value of the reactivity of the law $\xi=k / V$, the entire dynamics can be pictured in a single phase portrait in the plane $(\rho, \alpha)$. This is illustrated in figure 9 , for a positive $k$ and a moderate (figure $9(\mathrm{a})$ ) and a high (figure $9(\mathrm{~b})$ ) reactivity set to $\xi=50$ and $\xi=500$ respectively. These portraits show the trajectories of the object in the mobile frame of the sensor whose detection range (fixed to one and a half times that of the total sensor length) is drawn with a dashed green line. It may be seem that any sphere initially located within the cone drawn with a dashed green line, termed the attraction cone, is steered to the head. Furthermore, increasing $\xi$ increases the reactivity of the attraction and the aperture of the attraction cone. Note that for high values of reactivity, the spherical object first moves to a straight line in the longitudinal axis of the sensor and then flows along it toward its head. In the inertial frame, this means that the sensor directs its head toward the sphere's center before going forward to reach it. This can be easily explained using the analytical model of lateral currents (18) restricted to the isotropic component of the object response. In fact, (24) steers the probe in order to ensure that $\delta I_{\text {lat } 3}=\delta \bar{I}_{\text {lat } 3}=0$. Then, noting that $\rho_{i} \sin \alpha_{i}=\rho \sin \alpha \forall i$ in expression (20) of $\delta \bar{I}_{\text {lat } 3}$, shows that increasing $\xi$ makes the closed loop dynamics converge more and more rapidly toward the asymptotic branch defined by $\alpha=0$. Note that this branch is defined by the root locus of $\delta \bar{I}_{\text {lat } 3}(\rho, \alpha)=0$, whose point singularities due to the $\rho$-dependency, are systematically crossed by forcing the condition $V=V_{d}$ of (24).

Now, in the case of an ellipsoidal object, the closed loop dynamics take the more general form $x^{\prime}=f(\xi, x)$ with $x=(\rho, \alpha, \theta)$, since they also depend on the orientation $\theta$ of the anisotropic object. In spite of this major difference, the attractive behavior is preserved as illustrated in figure 10(a) which displays a typical trajectory around the test ellipsoid in the probe frame controlled by (24). Nevertheless the asymptotic branch along which the probe converged toward a small sphere now has two branches symmetrically tilted with respect to the probe axis, as shown in figure 11 . As in the case of the sphere these two branches are analytically defined as the root locus of $\delta I_{\text {lat } 3}=\delta \bar{I}_{\text {lat } 3}+\delta \hat{I}_{\text {lat } 3}=0$, which now depend on both $\alpha$ and $\theta$ through the anisotropic component $\delta \hat{I}_{\text {lat } 3}$. For sufficiently high values of $\xi$, the control ensures $\delta I_{\text {lat } 3} \simeq 0$, and (4) shows that after reaching one of the two branches (between positions $A$ and $B$ in figure 10(a)), the angles $\alpha$ and $\theta$ evolve along the branches (between positions $B$ and 


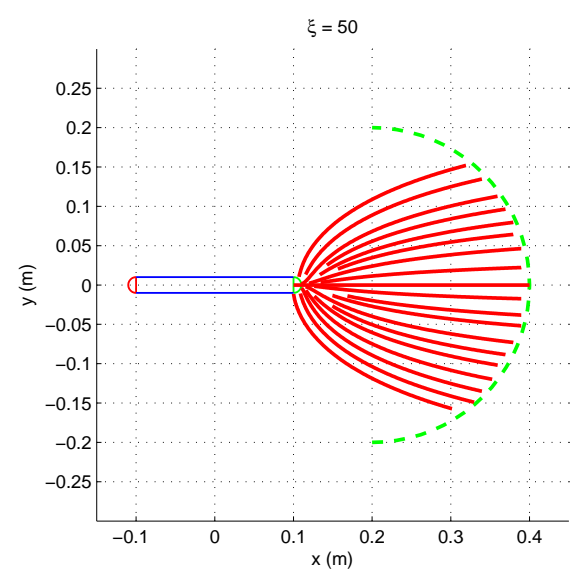

(a)

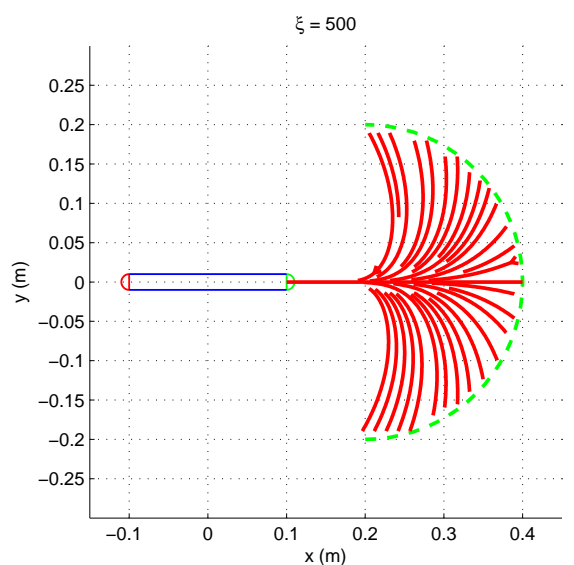

(b)

Figure 9. Portrait of the trajectories of a sphere in the reference frame of the sensor when it is controlled by the attractive version of (24), (a) for $\xi=50$, and (b) $\xi=500$.

$C$ of figure 10(a)) while ensuring $\alpha^{\prime}+\theta^{\prime}=0$. Note also that the $\theta$-dependency slightly affects the orientation of the branches as illustrated in figure 10(b), where several object trajectories are drawn depending on the orientation $\theta+\alpha$ of the ellipsoid with respect to the sensor axis at the initial instant of the detection.

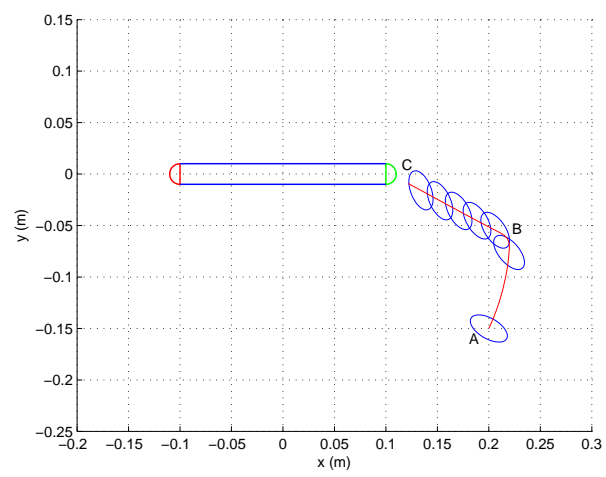

(a)

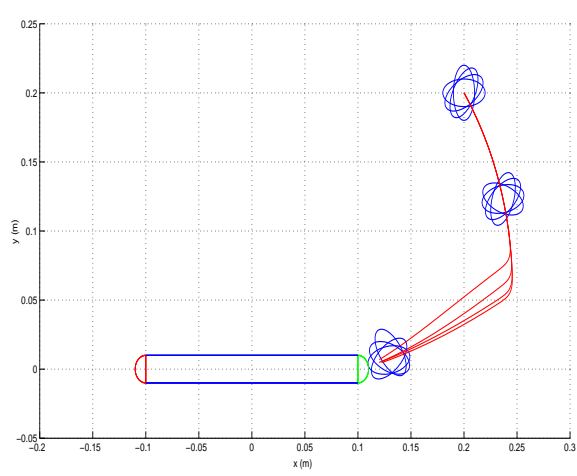

(b)

Figure 10. Illustration of the trajectories of an ellipsoid in the sensor frame when it is controlled by the attractive version of (24) for a high reactivity $(\xi=500)$, (a) and an initial tilt angle $\alpha+\theta=-30^{\circ}$. (b) Influence of the object tilt angle at the detection time. The object trajectory is computed with the same reactivity and an initial tilt angle $\alpha+\theta=0,30^{\circ}, 60^{\circ}, 90^{\circ}(\mathrm{b})$.

As expected, this $\theta$-dependency does not affect the overall attractive behavior as it is illustrated in figure 11(a) and 11(b), which respectively show the portrait of trajectories at detection time: $\theta+\alpha=0^{\circ}$, and $\theta+\alpha=30^{\circ}$. 


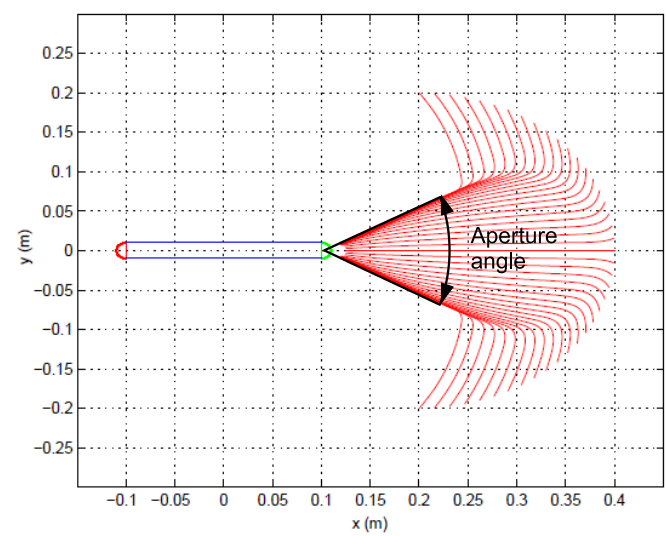

(a)

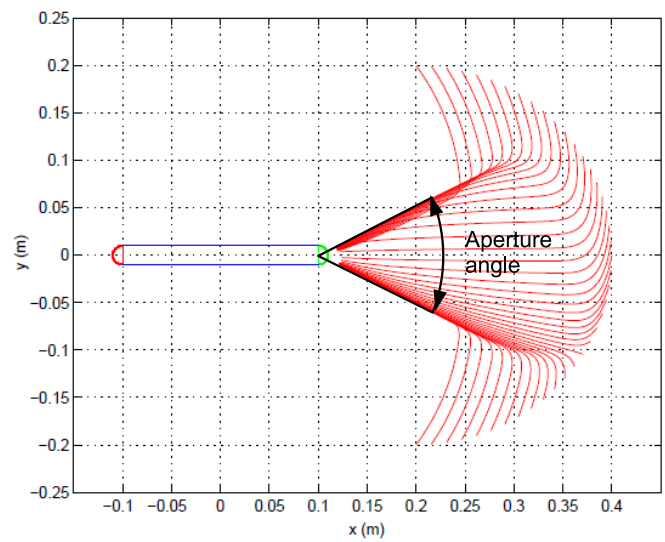

(b)

Figure 11. Portrait of trajectories of an ellipsoid in the sensor frame with $\xi=500$ and (a) a zero initial tilt angle. (b) As in (a) but with an initial tilt angle of $30^{\circ}$.

Note that the aperture angle between the two symmetric asymptotic branches only depends on the anisotropic component of the object's response and that this angle does decrease with the magnitude of the anisotropic response. This is shown in figure 12 which plots the dependency of this aperture angle as a function of $\hat{\lambda}$, or equivalently, of the ellipsoid aspect ratio $a / b$.

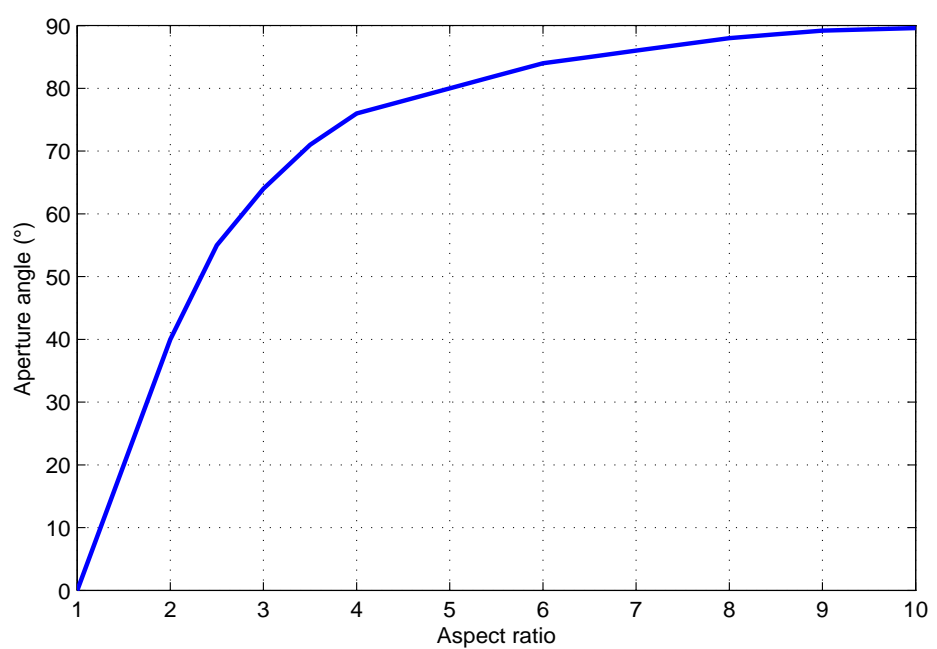

Figure 12. Aperture angle of the reactive attractive law as a function of the object aspect ratio.

\subsection{First transition: stop while facing the object}

Returning to the global strategy illustrated in figure 7 , we consider our probe to be in the same condition as the previous phase, i.e. it approaches the object to headfirst. We now consider the transition leading to the probe stopping near the 
object. Ideally, we would like to find a condition of the measured currents which, when satisfied, would ensure the probe stops at a fixed $\rho$, independent of the incidence angle $\alpha$ at which the probe discovers the object, and of the $\alpha+\theta$ orientation of the object with respect to the probe. Such a condition could be encoded into a function of the measurements capable of imaging depth perception along the probe's length as it approaches the object. To introduce such a function, we make use of the axial currents, which depend only on depth [Boyer et al., 2012], and reconsider the expression (10) of $\phi_{a}$ that once inserted into $\boldsymbol{\Phi}_{a}=\left(\phi_{a}\left(\mathbf{x}_{1}\right), \phi_{a}\left(\mathbf{x}_{2}\right), \phi_{a}\left(\mathbf{x}_{3}\right)\right)^{T}$ of $(1)$, can be set in the following matrix form:

$$
\delta \mathbf{I}_{a x}=\mathbf{C}^{(0)}\left[\begin{array}{l}
\phi_{1}\left(\mathbf{x}_{1}\right) \\
\phi_{1}\left(\mathbf{x}_{2}\right) \\
\phi_{1}\left(\mathbf{x}_{3}\right)
\end{array}\right]=\left(\begin{array}{ccc}
C_{11}^{(0)} & C_{12}^{(0)} & C_{13}^{(0)} \\
C_{21}^{(0)} & C_{22}^{(0)} & C_{23}^{(0)} \\
C_{31}^{(0)} & C_{32}^{(0)} & C_{33}^{(0)}
\end{array}\right)\left[\begin{array}{c}
\frac{\boldsymbol{\rho}_{1} \cdot \mathbf{p}}{\left\|\boldsymbol{\rho}_{1}\right\|^{3}} \\
\frac{\boldsymbol{\rho}_{2} \cdot \mathbf{p}}{\left\|\boldsymbol{\rho}_{2}\right\|^{3}} \\
\frac{\boldsymbol{\rho}_{3} \cdot \mathbf{p}}{\left\|\boldsymbol{\rho}_{3}\right\|^{3}}
\end{array}\right]
$$

from which we extract the axial currents flowing across the neck electrode:

$$
\delta I_{a x 2}=\left(C_{21}^{(0)} \frac{\boldsymbol{\rho}_{1}}{\rho_{1}^{3}}+C_{22}^{(0)} \frac{\boldsymbol{\rho}_{2}}{\rho_{2}^{3}}+C_{23}^{(0)} \frac{\boldsymbol{\rho}_{3}}{\rho_{3}^{3}}\right) \cdot \mathbf{p} \triangleq \mathbf{C}_{2} \cdot \mathbf{p} .
$$

Due to the conservation of currents, the conductivity matrix of the sensor when no object is nearby, satisfies the relation $C_{21}^{(0)}+C_{22}^{(0)}+C_{23}^{(0)}=0$ with three commensurable $C_{2 i}^{(0)}$ (see (2)). Thus, in the general case where $\alpha \neq 0$, the factors $\boldsymbol{\rho}_{i} /\left\|\boldsymbol{\rho}_{i}\right\|^{3}$ in (26), define at each moment in time a basis of three commensurable vectors of $\mathbb{R}^{3}$, so one can expect that, when the sensor approaches an object, a reasonable position $(\rho, \alpha)$ of the object such that $\mathbf{C}_{2}=\mathbf{0}, \mathbf{C}_{2}(\rho, \alpha)$ denoting a $3 \times 1$ vector-function defined in $(26)$, always exists. Of course, when $\mathbf{C}_{2}=\mathbf{0}, \delta I_{a x 2}$ changes sign by crossing $\delta I_{a x 2}=0$. The idea implemented hereafter consists in stopping the sensor when $\delta I_{a x 2}$ changes sign, i.e., when the condition $\delta I_{a x 2}=0$ is satisfied. This may happen in two other circumstances (than $\mathbf{C}_{2}=\mathbf{0}$ ) namely when $\mathbf{p}=\mathbf{0}$ and $\mathbf{C}_{2} \perp \mathbf{p}$. The first circumstance is absurd (it means that there is no object in the scene), while the second is purely fortuitous (and has never been observed after numerous simulations and experiments). The condition $\delta I_{a x 2}=0$ can be considered as equivalent to $\mathbf{C}_{2}=\mathbf{0}$. Using (26), in which we insert the parametrization of figure 4 and the consequence of current conservation $C_{21}^{(0)}+C_{22}^{(0)}+C_{23}^{(0)}=0$, the condition $\mathbf{C}_{2}=\mathbf{0}$ can be detailed as:

$$
\left\{\begin{array}{l}
C_{23}^{(0)}\left(\frac{\rho}{\rho^{3}}-\frac{\rho+l \cos (\alpha)}{\|\boldsymbol{\rho}+\mathbf{l}\|^{3}}\right)+C_{22}^{(0)}\left(\frac{\rho+\Delta l \cos (\alpha)}{\|\boldsymbol{\rho}+\Delta \mathbf{l}\|^{3}}-\frac{\rho+l \cos (\alpha)}{\|\boldsymbol{\rho}+\mathbf{l}\|^{3}}\right)=0 \\
C_{23}^{(0)}\left(\frac{l \sin (\alpha)}{\|\boldsymbol{\rho}+\mathbf{l}\|^{3}}\right)+C_{22}^{(0)}\left(\frac{l \sin (\alpha)}{\|\boldsymbol{\rho}+\mathbf{l}\|^{3}}-\frac{\Delta l \sin (\alpha)}{\|\boldsymbol{\rho}+\Delta \mathbf{l}\|^{3}}\right)=0
\end{array}\right.
$$

where $\mathbf{l}=l \mathbf{e}_{\|}$and $\Delta \mathbf{l}=\Delta l \mathbf{e}_{\|}$. The two equations (27) define a non-linear algebraic system whose roots $(\rho, \alpha)$ are the positions of the object in the sensor frame for which $\delta I_{a x 2}=0$. 


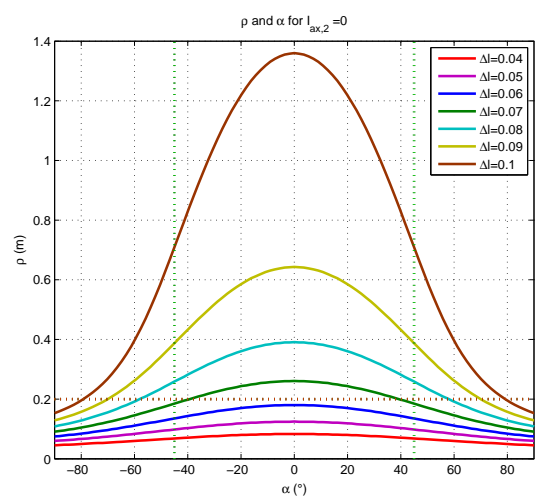

(a)

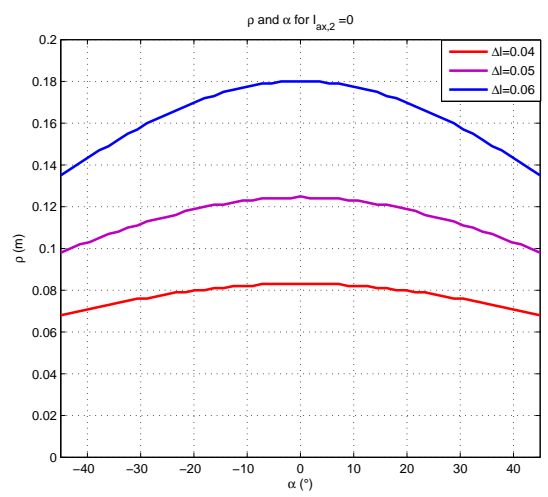

(b)

Figure 13. (a) Stopping distance $\rho$ for a sensor of various values of $\Delta l$ as a function of $\alpha$. Dashed lines show the limits of the sensor range and the aperture cone of the reactive attractive law (24), for an ellipsoid of maximal aspect ratio of 2. (b) Same, but magnified view of area of interest.

The root loci of the system (27) are drawn in figure 13(a) for various distances $\Delta l$ between the head and the neck electrodes. These plots show that for any $\Delta l$ there always exists a set of $(\rho, \alpha)$ defined by a bell shaped curve such that $\delta I_{a x 2}=0$. The different object positions strongly depend on the design variable $\Delta l$ (shifting $\Delta l$ from $0.09 \mathrm{~m}$ to $0.1 \mathrm{~m}$ doubles the $\rho$ distance at a fixed $\alpha=0$ ). Let us also note that $\rho$ weakly depends on $\alpha$ for reasonably low values of $\Delta l$, a situation reinforced by the fact that the domain of possible root locus is in reality restricted by the sensor range and the cone aperture angle imposed by the reactive law outlined in section 6. Figure 13(b) displays the root loci of (27) in a more realistic sub-domain for ellipsoids of aspect ratios between 1 and 5, and a high reactivity of the attractive law of phase 1 (note that in this case, the incident angle at which the probe discovers the object is dependent on the ellipsoid aspect ratio as in the plot in figure 12).

\subsection{Second phase and second transition: object clearance}

We now consider the second phase and the transition that inhibits it (see algorithm in figure 7). They both define a single behavior in which the sensor clears the object after having found it (phase 1) and has stopped near it (transition 1). This behavior is obtained by applying the reactive control law (24) but in its repulsive variant, i.e. with:

$$
V=0, \text { and: } \Omega=-k \frac{\delta I_{l a t 3}}{\left|\delta I_{a x 3}\right|} .
$$

Compared to $(24)$, we reverse the sign of the steering gain $(k>0)$ to ensure the sensor turns its head away from the object. This is performed with $V=V_{d}=0$, maintaining the distance between the probe and object centers constant while manoeuvering. While performing this probe rotation, the head axial current $I_{a x 3}=I_{3}^{(0)}+\delta I_{a x 3}$ undergoes a stereotyped time-evolution which we will now analyse further in order to define a 
condition in which the probe stops its rotation while remaining clear of the object. Note that, from (1) and (21), we can rewrite the model of axial currents in the matrix form:

$$
\mathbf{I}_{a x}=\left[\mathbf{C}^{(0)}-\left(\mathbf{C}^{(0)} \frac{G P G^{\mathrm{T}}}{4 \pi \gamma_{o}} \mathbf{C}^{(0)}\right)\right]\left(\begin{array}{l}
1 \\
0 \\
0
\end{array}\right) U \triangleq \mathbf{C}\left(\begin{array}{l}
1 \\
0 \\
0
\end{array}\right) U,
$$

where $\mathbf{C}$ defines the conductivity offered by the scene, while $G$ is a $3 \times 3$ matrix defined as:

$$
G=\left(\begin{array}{ccc}
\frac{\cos \left(\alpha_{1}\right)}{\rho_{1}^{2}} & \frac{\sin \left(\alpha_{1}\right)}{\rho_{1}^{2}} & 0 \\
\frac{\cos \left(\alpha_{2}\right)}{\rho_{2}^{2}} & \frac{\sin \left(\alpha_{2}\right)}{\rho_{2}^{2}} & 0 \\
\frac{\cos \left(\alpha_{3}\right)}{\rho_{3}^{2}} & \frac{\sin \left(\alpha_{3}\right)}{\rho_{3}^{2}} & 0
\end{array}\right) .
$$

In (30), the third column is zero because the scene is symmetrical in the horizontal plane. Note also that $\rho_{3}=\rho$ and $\rho_{i} \sin \left(\alpha_{i}\right)=\rho \sin (\alpha)$ for any $i$. Moreover, as described in [Servagent et al., 2013], we have the following relation at leading order (with respect to the object volume):

$$
\mathbf{C}=\mathbf{C}^{(0)}-\mathbf{C}^{(0)} \frac{G P G^{\mathrm{T}}}{4 \pi \gamma_{o}} \mathbf{C}^{(0)} \simeq\left(\mathbf{R}^{(0)}+\frac{G P G^{\mathrm{T}}}{4 \pi \gamma_{o}}\right)^{-1}
$$

where $\mathbf{R}^{(0)}$ is the resistance matrix of the sensor immersed in a scene with no object, while the matrix $\left(4 \pi \gamma_{o}\right)^{-1} G P G^{T}$ represents the perturbation of the resistivity of the scene offered to the probe due to the presence of the object. Based on this insight, the axial current $I_{a x 3}=(0,0,1) \mathbf{I}_{a x}$ is an image of the resistance of the scene between the tail-emitter $\mathcal{E}_{1}$ and the head-electrode $\mathcal{E}_{3}$. As such, $I_{a x 3}$ encodes not only the intrinsic features of the object (material, shape, size) and its tilt angle through $P$, but also its position in the sensor frame through the $G$ matrix of (29). In particular, due to the factors $\rho_{i}^{-2}$ in (30), this current increases (respectively decreases) when one of the two tip electrodes approaches a conductive (respectively insulating) object. Reciprocally, $I_{a x 3}$ decreases (respectively increases) when one of the two tip electrodes moves away from a conductive (respectively insulating) object. All of these trends reflecting the variations in the scene resistance between the two tip electrodes $\mathcal{E}_{1}$ and $\mathcal{E}_{3}$. This is illustrated in figure 14(b) which shows the simulated evolution of $I_{a x 3}$ as a function of the tilt angle of the probe (denoted $\mu$ ) for our conductive ellipsoidal test object with a fixed center, but different orientations of its axis in the horizontal plane (of the motion). The evolution starts from a probe pose in which the head points toward the object center (see figure $14(\mathrm{a}))$. 


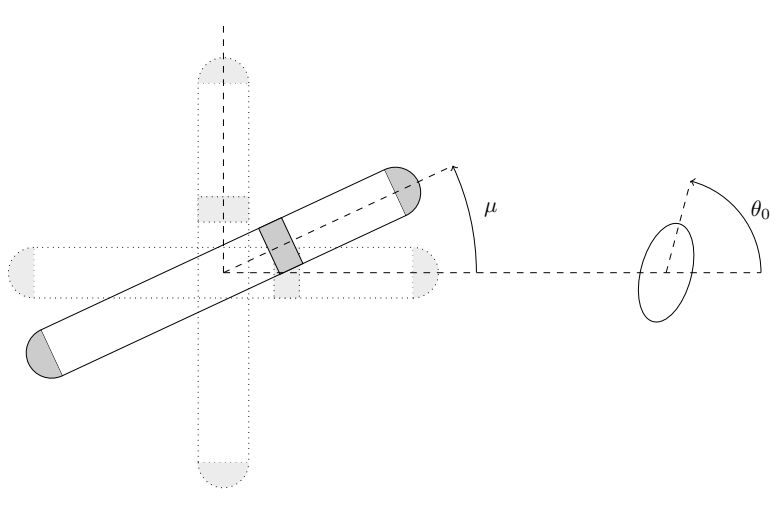

(a)

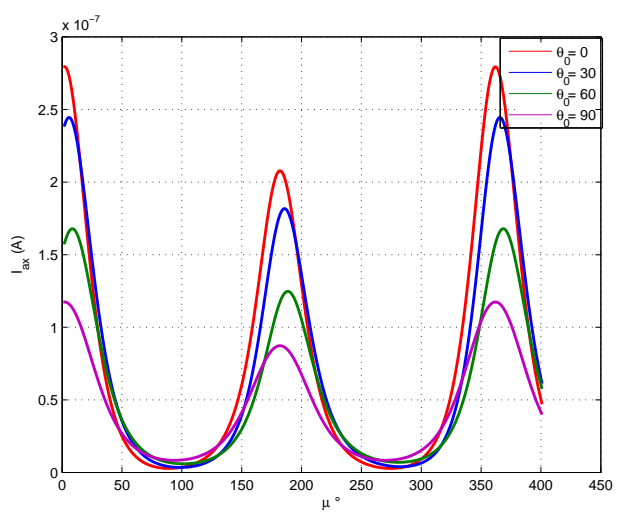

(b)

Figure 14. Evolution of $I_{a x 3}$ as a function of the tilt angle of the probe $\mu$ when the probe initially points toward the object center. (a) Condition of the experimental test. (b) Evolution of $I_{a x 3}$ for an ellipsoid $(a=2, b=1)$ tilted at different angles $\left(0^{\circ}, 30^{\circ}\right.$, $60^{\circ}$ and $90^{\circ}$ ).

As expected, as rotation is initialised, the sensor head is at its closest to the conductive object, so the resistance is minimal and the axial current is maximal. Furthermore, while rotating, the same situation obtains each time one of the two tips (head/tail) of the probe is closest to the object, i.e., at every multiple of $180^{\circ}$. The odd multiples correspond to the case where the tail faces the object, which generates a local maximum of $I_{a x 3}$. The even multiples repeat the starting pose in which the head faces the object while a global maximum of $I_{a x 3}$ is reached. Furthermore, between each of its maximums, $I_{a x 3}$ reaches a minimum value which corresponds to a pose in which the sensor is electrically as far as possible from the object ( $V$ being fixed to zero). These observations hold perfectly when the test ellipsoid has one of its axe of symmetry aligned with the probe axis at the initiation of the phase. This is illustrated in figure 14 where the red and purple plots correspond to an object initially tilted at angles of $\theta_{0}=0^{\circ}$ and $\theta_{0}=90^{\circ}$ respectively, and show that $I_{a x 3}$ does exhibit a maximum at $\mu=0^{\circ}$, and reaches a minimum when $\mu=90^{\circ}$. However, rotating the ellipsoid to an arbitrary initial angle $\theta_{0}$ introduces an asymmetry in the initial scene that shifts the maxima and minima of a tilt offset which does not exceed $\pm 15^{\circ}$ (see the other plots obtained when the ellipsoid is rotated of $30^{\circ}$ and $60^{\circ}$ ). For a resistive object, the evolution of $I_{a x 3}$ as a function of the tilt angle of the probe $\mu$, is similar but reversed with respect to the $I_{a x 3}=0$ axis. Hence, in both cases (conductive or resistive) $\left|I_{a x 3}\right|$ cyclically and alternately reaches local maxima and minima in the same poses of the probe. In the context of our global strategy, when $\left|I_{a x 3}\right|$ reaches its first minimum (after the activation of the law (28)), we switch off the repulsive law while memorizing the axial current $I_{a x 3}$ and activate the subsequent and final phase (numbered 3 in the algorithm in figure 7). 

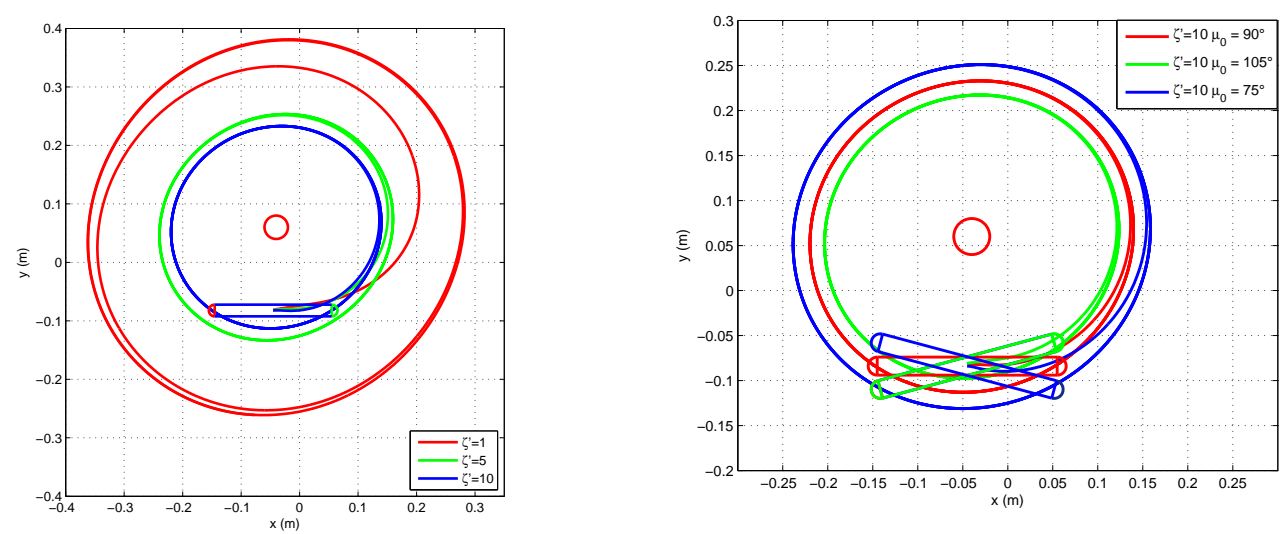

Figure 15. (a) Paths of the sensor for different values of reactivity $\xi^{\prime}=k^{\prime} / V$ starting from a probe pose perpendicular to the line between the probe and object centers. (b) Paths of the probe revolving around a sphere of high reactivity, starting from different tilted pose of the probe.

\subsection{Third phase: following the object boundaries}

In this subsection, we analyse the behavior "Following the boundaries of an electrically contrasted object". This basic behavior is achieved through the simple sensor-based feedback law:

$$
V=V_{d}, \text { and } \Omega=k^{\prime}\left(I_{a x 3}-I_{a x 3}^{d}\right),
$$

where $V_{d}$ is a constant positive value ensuring the probe moves forward with a constant axial velocity $V_{d} \mathbf{e}_{\|}$and $k^{\prime}$ is a second steering gain (after that of $(24,28)$ ) which makes the probe track an iso-value $I_{a x 3}=I_{a x 3}^{d}$, where $I_{a x 3}^{d}$ is the value of $I_{a x 3}$ memorized at the end of the previous phase, i.e. when $\left|I_{a x 3}\right|$ reaches its first minimum after activation of the repulsion. Using the model developed in the previous section, we know that $I_{a x 3}$ images the position of the object in the sensor frame. Moreover, figure 14 shows that the minimum values of $I_{a x 3}$ are weakly affected by the tilt angle of the ellipsoid.

As a result, imposing $I_{a x 3}=I_{a x 3}^{d}$ through the control law (32) with $I_{a x 3}^{d}$ being the minimum values measured in similar conditions to those of figure 14, should maintain the object in the same position in the mobile sensor frame. In other words, the probe moves forward at a constant linear velocity $V_{d}$, while tilting its body to maintain $I_{a x 3}=I_{a x 3}^{d}$. It thus revolves around the object at a nearly constant distance from it. This basic idea is confirmed by simulation of the closed loop dynamics $x^{\prime}=f(\xi, x)$ obtained by inserting (32) into the kinematic model of the scene (4). In figure 15(a) we plotted the paths of the probe starting in a pose perpendicular to the line linking the center of the probe with a spherical object, i.e. $\mu(t=0)=90^{\circ}$ for different values of reactivity $\xi^{\prime}=k^{\prime} / V$. In figure $15(\mathrm{~b})$, the same numerical simulations were performed but with an initial tilt offset of $\pm 15^{\circ}$ with respect to the perpendicular configuration. Such an offset takes into account the slight variations of the minimum detection occurring at 
the end of the previous (clearance) phase. This control law has been tested in many experiments in the test bed described in section 3 with objects of different shape, size and materials. We report two representative tests in which the sensor starts from a given initial pose $A$, then moves in straight line while at a given instant (illustrated by pose $B$ on these plots), the axial current $I_{a x 3}$ is memorized and imposed as $I_{a x 3}^{d}$ in the reactive law (32) which is applied at all further instants. This simple test is illustrated for a conductive ellipsoid and cube in figures 16 and 17 respectively. In both cases, the plotted probe trajectory shows that after switching from open loop to closed loop control (32), the sensor revolves around the object in order to maintain the prescribed memorized value of $I_{a x 3}$.
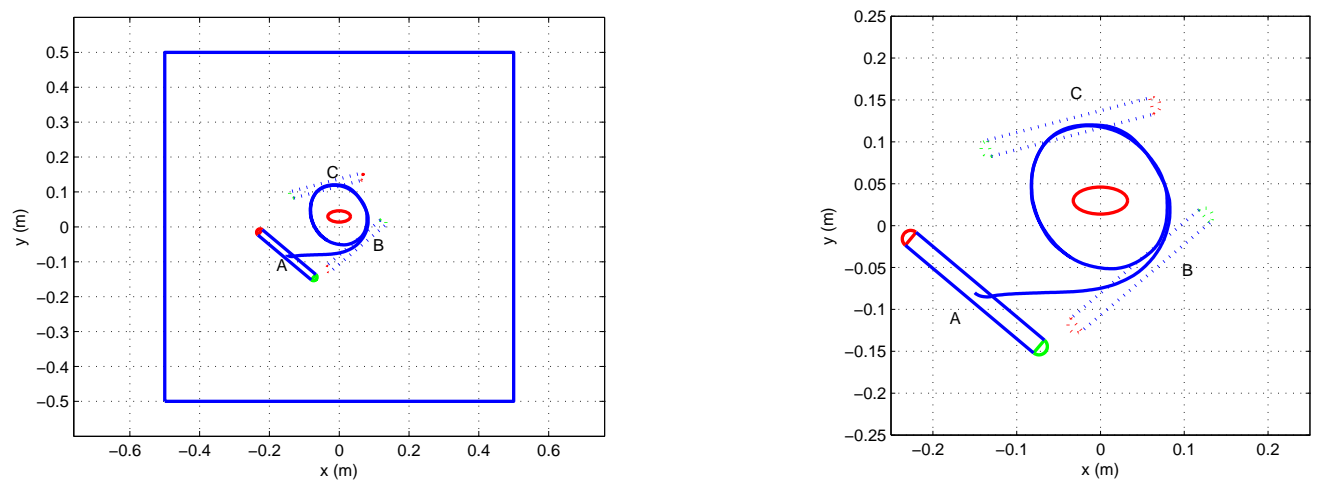

Figure 16. Sensor controlled by the reactive law (32) in the presence of a conductive ellipsoid. (a) The law is activated from pose $B$ to $C$. View from the top of the tank. (b) Magnified view of the path around the object.
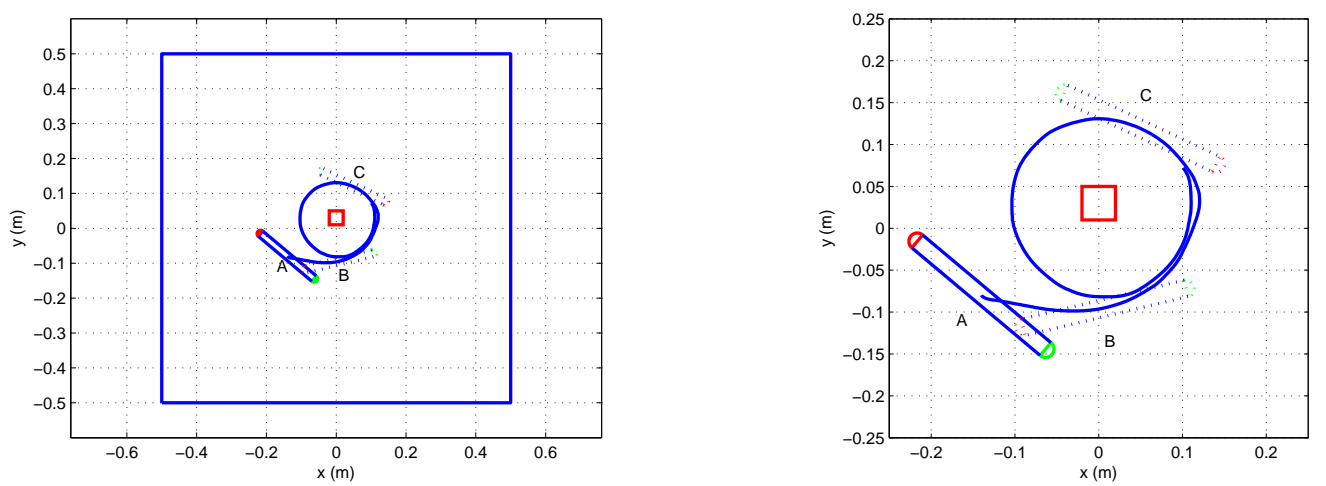

Figure 17. Sensor controlled by the reactive law (32) in the presence of a conductive cube. (a) The law is activated from the pose $B$ to $C$. View from the top of the tank. (b)Magnified view of the path around the object. 


\section{Experimental tests of the overall strategy}

We report here some of the experimental results obtained by applying the above object inspection control strategy. The experimental conditions are those described in section 3. Two types of objects are inspected. The first is an insulating cube, the second is a combination of large flat insulating objects (finite walls). The electric feedback loops (24) and (32) are tuned once for all with $k=50 \mathrm{~V}$ and $k^{\prime}=2.5 \mathrm{~V}$ respectively, and then applied to all the tests. Figures 18 (top) and 19 (top) display the motion path of the sensor for the inspection strategy. As expected, for each experiment the sensor first starts by seeking the electrically contrasted objects. After having found one, it stops while facing it (in pose $B$ ), retreats from its electric influence through a clearance rotating phase, and then follows the boundaries of the object starting from pose $B^{\prime}$. For the smooth objects, the path around them nearly follows their boundaries (a circle for the sphere, an ellipse for the ellipsoidal object). Figure 18 shows a nearly circular sensor path centered on the insulating cube located in the middle of the tank, with two orientations $0^{\circ}$ and $45^{\circ}$ with respect to the initial position of the sensor's axis. This can be explained by the fact that the electric responses of a sphere and that of a cube are very similar, and in fact indistinguishable at the leading order. However, this basic property is only true for small objects. Thus, due to the largeness of our test-cube, the sensor trajectory is not a regular circle. These distortions of the circular path map the sharp edges of the cube. The time-evolution of the currents $\delta I_{a x 2}$ and $\delta I_{a x 3}$ is plotted in figure 18 (bottom) for the cube (similar plots are observed for the sphere and the ellipsoid). As expected, phase 2 (clearance from pose $B$ to pose $B^{\prime}$ ) is activated by the change of sign of $\delta I_{a x 2}$ while phase 3 (revolution after pose $\left.B^{\prime}\right)$ is activated when $\left|I_{a x 3}\right|=\left|I_{a x 3}^{(0)}+\delta I_{a x 3}\right|$ reaches its minimum value. In this circumstance the value of $I_{a x 3}$, indicated by the horizontal grey dashed line, is memorized to inform the reactive orbiting law (32).

Finally, the case of the inspection of large objects is illustrated in the two last examples in figure 19. In the first example, the object is an insulating wall placed in the middle of the tank. In the second example, there is no object in the tank except its 4 insulating vertical walls and a removable small insulating plane initially placed at the bottom left corner of the tank. In the first (respectively the second) case, after having stopped in front of the center wall (respectively the right wall), the sensor orbits around the center wall (respectively the tank's boundaries). In the first case, we plotted several paths obtained using the overall strategy with different initial poses. In the second case, the strategy is initiated with the small removable wall at the left-bottom corner, and after one revolution around the tank, this small panel is suddenly removed. When moving past this corner, the sensor re-adjusts its path (indicated by the dashed line with the removable panel in the tank and in continuous line, after it was removed) to follow the two original fixed walls of the tank. Figure 19 (bottom) shows the time evolution of the measured currents along the sensor trajectory when it is following the tank walls. The two time transitions of the sequential graph $(7(\mathrm{a}))$ are indicated by 
poses $B$ and $B^{\prime}$ as in figure $(7(\mathrm{~b}))$. Note how the first transition (corresponding to pose $B)$ is activated when the axial current $\delta I_{a x 2}$ crosses the zero value, while the second transition (corresponding to pose $B^{\prime}$ ) is activated when $\left|I_{a x 3}\right|=\left|I_{a x 3}^{(0)}+\delta I_{a x 3}\right|$ reaches its minimum value, the circumstance in which the current $I_{a x 3}$, indicated by the dashed grey horizontal line, is memorized.
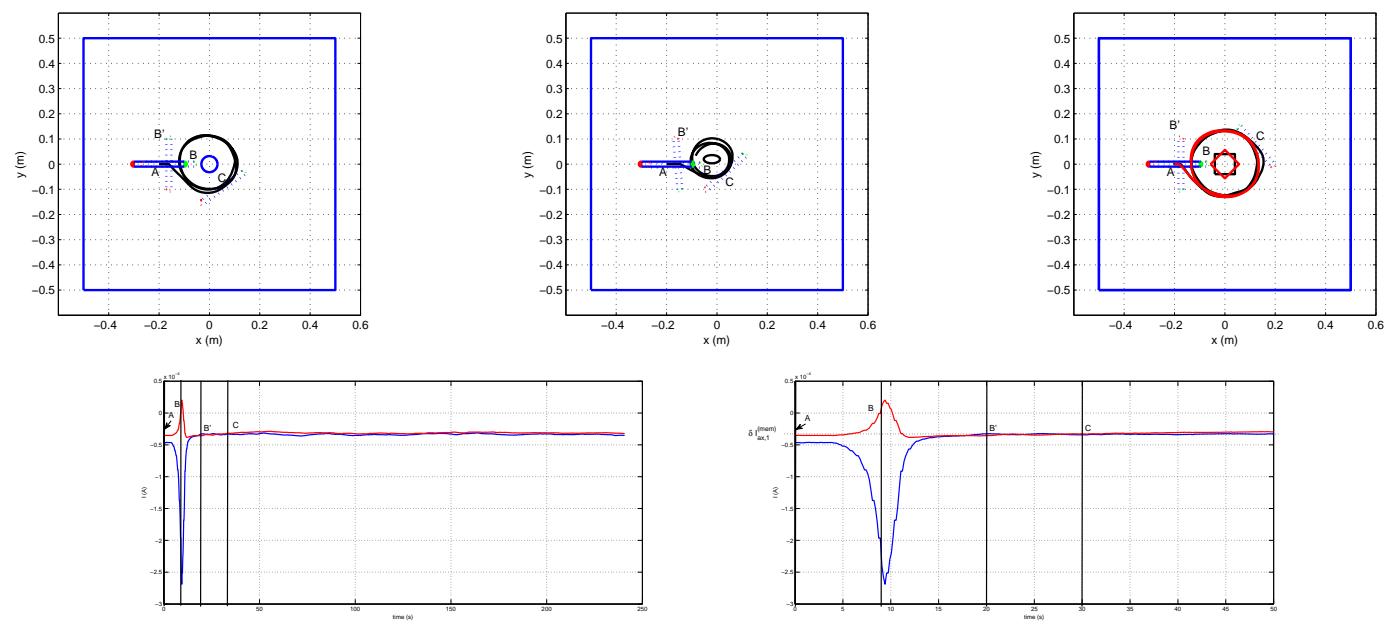

Figure 18. Inspection of the boundaries of an insulating sphere, ellipse and cube. (bottom - left)) Time evolution of the currents $\delta I_{a x 3}$ (blue) and $\delta I_{a x 2}$ (red) during inspection of the cube; (right) detailed view of (c) highlighting a phase transition.

\section{Conclusion and perspectives}

In this article we have addressed the problem of object inspection using a sensor inspired by electric fish. The solution enables to seek any electrically contrasted object and then to orbit around its boundaries. Starting from the memory-less reactive steering strategy dedicated to object seeking and/or obstacle avoidance initially presented in [Boyer et al., 2013] for a "binocular" sensor with two left-right head receptors, we progressively increased the number of electrodes and provided our controller with memory in order to perform object inspection. This minimal approach enabled clear identification of the key physical principles required for these control objectives. In particular, compared to our previous work on reactive navigation, the object inspection requires that our sensor performs a depth measurement while facing the object. This additional capability requires an additional neck electrode whose axial (left + right) perturbing current $\delta I_{a x 2}=I_{a x 2}-I_{2}^{(0)}$ enables this perceptual feature. In the context of underwater robotics, this control approach could be used for the autonomous navigation of an AUV in turbid waters and muddy environments, for instance to collect data that could subsequently be processed off-line to build a model of objects. Remarkably, the approach does not require any model of the electric interactions between the sensor and the surrounding objects. According to the concept of embodied intelligence 

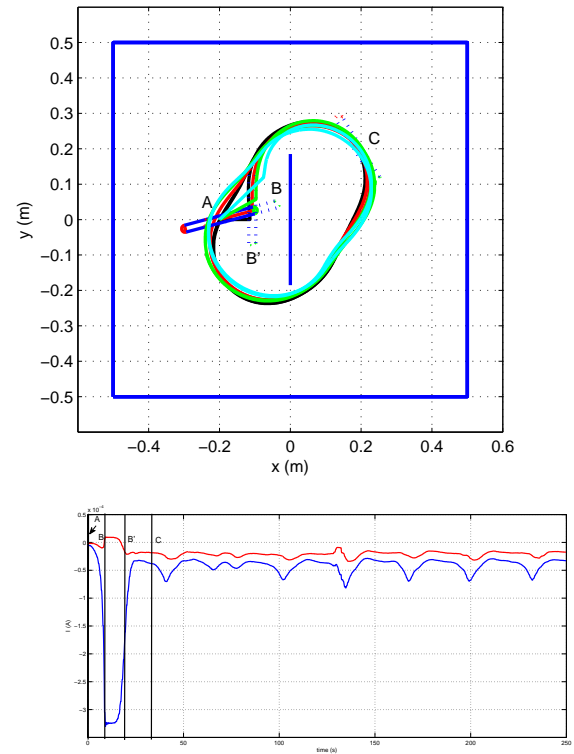
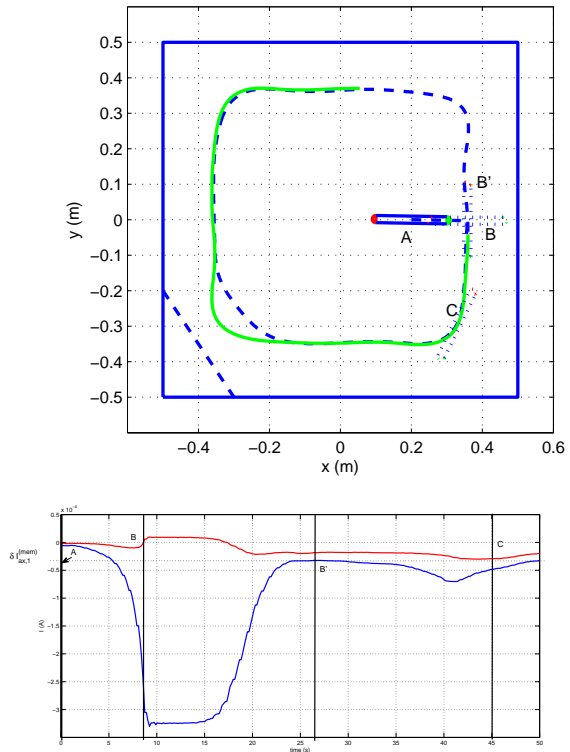

Figure 19. Inspection of large objects: (top) An insulating wall is placed in the tank center (left); removable corner (right). (bottom) Time evolution of the currents $\delta I_{a x 3}$ (blue) and $\delta I_{a x 2}$ (red) along the sensor path when the sensor follows the walls of the tank: (left) complete time evolution; (right) detailed view of (a) highlighting a phase transition.

[Pfeifer et al., 2007], the model is in fact totally embodied into the morphology of the sensor and the physics (electrokinetics of perception and kinematics of locomotion) of its interactions with its environment (see section 6.2). In this respect, note that the bi-lateral (left-right) symmetry commonly observed in most of animals, and electric fish in particular, is a key feature for steering the robot with the reactive law (24). The additional morphological parameter $\Delta l$ (the distance between the head and neck electrodes) is another key parameter which totally encodes the stopping pose illustrated in figure 13(a). This approach allows the same behavioral inspection strategy to be obtained in all circumstances, particularly when there is no prior model of the object. This situation is often encountered, and represents remarkable advantages since, except for small ellipsoidal objects or infinite walls and corners, we only have numerical finite element models which are unusable in real time robotics applications. Beyond the empirical scenarios reported in the article, the insights developed here open promising perspectives of accurate sensor positioning with respect to an object. For example in the stopping transition illustrated in section 6.3, at the end of phase 1, the sensor is aligned with one of the object's axis of symmetry, and so therefore it is pointing directly at its geometric center, the incident angle $\alpha$ being zero. In this simplified case, the condition $\delta I_{a x 2}=0$ defines an invariant distance to the object, i.e. a condition on the measurements only, and whose satisfaction ensures a fixed distance between the probe and the object, this distance being independent of the intrinsic object's properties. As a result, replacing phase 1 by active self-alignment 
of the probe along one axis of symmetry of the object (through feedback control), would allow the sensor to be positioned at a fixed distance from the object's center at transition 1, as is observed in fish when they perform the PMA 1 illustrated in figure 1. This strategy is currently being studied and will be presented in a future article.

\section{Acknowledgment}

This work was supported by the European Union, by funding the Project: EU H2020 FET-Proactive project 'subCULTron', no. 640967

\section{References.}

[Ammari and Kang, 2007] Ammari, H. and Kang, H. (2007). Polarization and moment tensors: with applications to inverse problems and effective medium theory, volume 162. Springer Science \& Business Media.

[Bai et al., 2012] Bai, Y., Snyder, J., Silverman, Y., Peshkin, M., and MacIver, M. (2012). Sensing capacitance of underwater objects in bio-inspired electrosense. In IEEE/RSJ Int. Conf. on Intelligent Robots and Systems.

[Boyer et al., 2012] Boyer, F., Gossiaux, P., Jawad, B., Lebastard, V., and Porez, M. (2012). Model for a sensor bio-inspired from electric fish. IEEE transactions on robotics, 28(2):492-505.

[Boyer and Lebastard, 2012] Boyer, F. and Lebastard, V. (2012). Exploration of objects by an underwater robot with electric sense. In Prescott, T., Lepora, N., Mura, A., and Verschure, P., editors, Biomimetic and Biohybrid Systems, volume 7375 of Lecture Notes in Computer Science, pages 50-61. Springer Berlin Heidelberg.

[Boyer et al., 2015] Boyer, F., Lebastard, V., Chevallereau, C., Mintchev, S., and Stefanini, C. (2015). Underwater navigation based on passive electric sense: New perspectives for underwater docking. The International Journal of Robotics Research, 34 (9):1228-1250.

[Boyer et al., 2013] Boyer, F., Lebastard, V., Chevallereau, C., and Servagent, N. (2013). Underwater reflex navigation in confined environment based on electric sense. IEEE Transactions on Robotics, 29(4):945-956.

[Caputi, 2004] Caputi, A. (2004). Contributions of electric fish to the understanding sensory processing by reafferent systems. Journal of Physiology-Paris, 98(1):81-97.

[Chevallereau et al., 2014] Chevallereau, C., Benachenhou, M., Lebastard, V., and Boyer, F. (2014). Electric sensor based control of underwater robot groups. IEEE Transactions on Robotics, 30(3):604-618.

[Hofmann et al., 2013] Hofmann, V., Sanguinetti-Scheck, J., Gómez-Sena, L., and Engelmann, J. (2013). From static electric images to electric flow: towards dynamic perceptual cues in active electroreception. Journal of Physiology-Paris, 107(1):95-106.

[Lane, 2012] Lane, D. (2012). Persistent autonomy artificial intelligence or biomimesis? In Autonomous Underwater Vehicles (AUV), 2012 IEEE/OES, pages 1-8.

[Lebastard et al., 2010] Lebastard, V., Chevallereau, C., Amrouche, A., Jawad, B., Girin, A., Boyer, F., and Gossiaux, P. (2010). Underwater robot navigation around a sphere using electrolocation sense and kalman filter. In IROS 2010 IEEE.

[Lissmann and Machin, 1958] Lissmann, H. and Machin, K. (1958). The mechanism of object location in gymnarchus niloticus and similar fish. The Journal of Experimental Biology, (35):451-486.

[MacIver et al., 2004] MacIver, M., Fontaine, E., and Burdick, J. (2004). Designing future underwater vehicles: principles and mechanisms of the weakly electric fish. IEEE Journal of Oceanic Engineering, pages 651-659. 
[MacIver and Solberg, 2001] MacIver, M. and Solberg, J. (2001). Towards a biorobotic electrosensory system. Autonomous robots, 11:263-266.

[Pfeifer et al., 2007] Pfeifer, R., Lungarella, M., and Lida, F. (2007). Self-organization, embodiment, and biologically inspired robotics. Science, 318(5853):1088-1093.

[Porez et al., 2011] Porez, M., Lebastard, V., Ijspeert, A., and Boyer, F. (2011). Multi-physics model of an electric fish-like robot: Numerical aspects anbd application to obstacle avoidance. In IEEE/RSJ Int. Conf. on Intelligent Robots and Systems, pages 1901-1906.

[Rasnow, 1996] Rasnow, B. (1996). The effects of simple objects on the electric field of apteronotus. Journal of Comparative Physiology A, 3(178):397-411.

[Servagent et al., 2013] Servagent, N., Jawad, B., Bouvier, S., Boyer, F., Girin, A., Gomez, F., Lebastard, V., and Gossiaux, P.-B. (2013). Electrolocation sensors in conducting water bio-inspired by electric fish. IEEE Sensor Journal, 13(5):1865-1882.

[Silverman et al., 2012] Silverman, Y., Snyder, J., Bai, Y., and MacIver, M. (2012). Location and orientation estimation with an electrosense robot. In IEEE/RSJ Int. Conf. on Intelligent Robots and Systems.

[Solberg et al., 2008] Solberg, J., Lynch, K., and MacIver, M. (2008). Active electrolocation for underwater target localization. The International Journal of Robotics Research, 27(5):529-548.

[Toerring and Belbenoit, 1979] Toerring, M. J. and Belbenoit, P. (1979). Motor programmes and electroreception in mormyrid fish. Behavioral Ecology and Sociobiology, 4(4):369-379.

[Toerring and Moller, 1984] Toerring, M.-J. and Moller, P. (1984). Locomotor and electric displays associated with electrolocation during exploratory behavior in mormyrid fish. Behavioural brain research, 12(3):291-306.

[Triantafyllou et al., 2000] Triantafyllou, M., Triantafyllou, G., and Yue, D. (2000). Hydrodynamics of fishlike swimming. Annual review of fluid mechanics, 32(1):33-53.

[von der Emde, 1999] von der Emde, G. (1999). Active electrolocation of objects in weakly electric fish. The Journal of Experimental Biology, 202:1205-1215.

[von der Emde et al., 2012] von der Emde, G., Gebhardt, K., and Behr, K. (2012). Non-visual orientation and communication by fishes using electrical fields: A model system for underwater robotics. In Robotics and Automation (ICRA), 2012 IEEE International Conference on, pages 1143-1148.

[von der Emde et al., 1998] von der Emde, G., Schwarz, S., Gomez, L., Budelli, R., and Grant, K. (1998). Electric fish measure distance in the dark. Letters to Nature, Nature, 395:890-894. 